\title{
The FRATS project: real-time searches for fast radio bursts and other fast transients with LOFAR at $135 \mathrm{MHz}$
}

S. ter Veen ${ }^{1,2}$, J. E. Enriquez ${ }^{2,3}$, H. Falcke ${ }^{2,1,4}$, J. P. Rachen², M. van den Akker², P. Schellart ${ }^{2,5}$, A. Bonardi², R. P. Breton ${ }^{6}$, J. W. Broderick ${ }^{1}$, S. Corbel ${ }^{7,8}$, A. Corstanje ${ }^{2}$, J. Eislöffel ${ }^{9}$, J.-M. Grießmeier ${ }^{10,8}$, J. R. Hörandel ${ }^{2,11,12}$, A. J. van der Horst ${ }^{13}$, C. J. Law ${ }^{14}$, J. van Leeuwen ${ }^{1,15}$, A. Nelles ${ }^{2,16}$, L. Rossetto ${ }^{2}$, A. Rowlinson ${ }^{15,1}$, T. Winchen ${ }^{17}$, and P. Zarka ${ }^{18,8}$

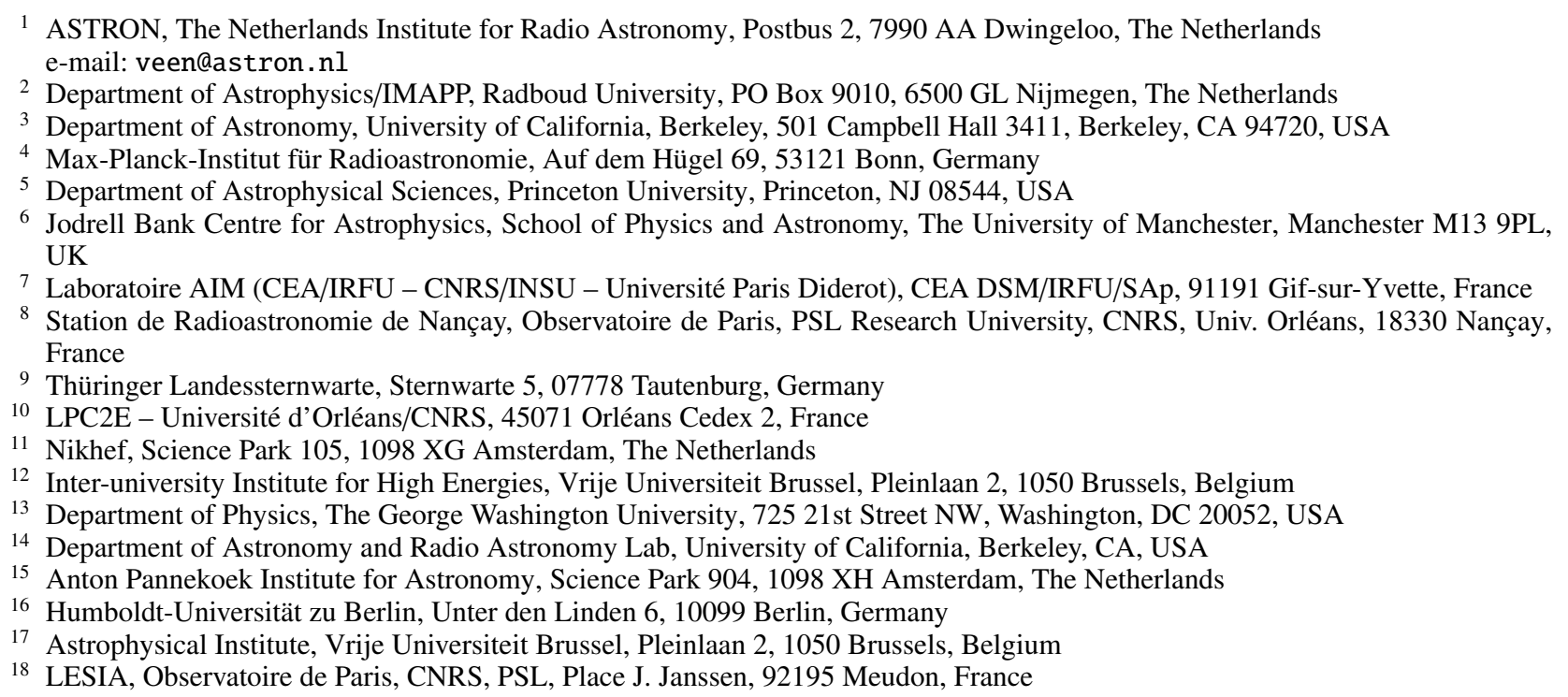

Received 21 December 2017 / Accepted 16 October 2018

\begin{abstract}
Context. In the previous decade, two new classes of fast radio transients were detected: the Galactic, rotating radio transients (RRATs) and the extragalactic fast radio bursts (FRBs). If the detectable emission of these objects extends to lower radio frequencies, the LOw Frequency ARray (LOFAR) is ideally suited to seek and localize these transients at frequencies of $10-250 \mathrm{MHz}$. This is due to LOFAR's sensitivity, diverse beamform capabilities, and transient buffers for the individual elements that allow post-event imaging of events, potentially at arcsecond resolution.

Aims. Our aim is to identify and localize pulses at frequencies below $250 \mathrm{MHz}$ and, in the case of nondetections, derive upper limits on the sky and volume rates of FRBs.

Methods. A real-time search program for fast radio transients is installed on the LOFAR systems which runs commensally with other observations, and uses the wide incoherent LOFAR beam $\left(11.25 \mathrm{deg}^{2}\right.$ at $\left.150 \mathrm{MHz}\right)$. Buffered data from hundreds of dipoles are used to reconstruct the direction and polarization information of the event, and to distinguish between celestial, terrestrial, and instrumental origins.

Results. Observations were taken covering either the frequency range $119-151 \mathrm{MHz}$ or in four frequency bands, each of $2 \mathrm{MHz}$ in width, centered at 124, 149, 156, and $185 \mathrm{MHz}$. A first pilot survey covered a range of dispersion measures (DM) below $120 \mathrm{pc} \mathrm{cm}^{-3}$, focusing on Galactic sources, and resulted in an upper limit on the transient rate at LOFAR frequencies of less than 1500 events per sky per day above a fluency of $1.6 \mathrm{kJy} \mathrm{ms}$ for an 8-ms pulse. A second pilot survey covered a range of DMs below $500 \mathrm{pc} \mathrm{cm}^{-3}$, focusing on extragalactic sources to about $1 \mathrm{Gpc}$, and resulted in an upper limit of 1400 events per sky per day above a fluency of $6.0 \mathrm{kJy} \mathrm{ms}$ for an $8-\mathrm{ms}$ pulse. Using a model for the distance-DM relationship, this equates to an upper limit of 134 events per Gpc $^{3}$ per day.
\end{abstract}

Key words. surveys - pulsars: general - instrumentation: interferometers - techniques: high angular resolution

\section{Introduction}

The study of fast radio transients, or subsecond dispersed pulses, is a field that has been rediscovered over the past decade. This has led to the identification of rotating radio transients
(RRATs, McLaughlin et al. 2006), pulsars that are more easily detectable through their individual pulses than by their average emission profile (Burke-Spolaor et al. 2013), and fast radio bursts (FRBs; Lorimer et al. 2007; Thornton et al. 2013), that are highly dispersed pulses from an extragalactic origin 
(Tendulkar et al. 2017). For both of these source classes, localization is an important part of unraveling the nature of their emission. For RRATs in fields with many high-energy sources, a precise localization is important for determining the X-ray or gamma-ray source to which they belong (Burke-Spolaor et al. 2013). Fast radio bursts could provide a probe to study the intergalactic medium, for example to identify missing baryons (Deng \& Zhang 2014; McQuinn 2014). For this, a host galaxy and corresponding redshift need to be determined. Localizing FRBs to within a host-galaxy can help us understand what sources could be responsible for the emission itself (Bassa et al. 2017). Additional information may come from observing FRBs and RRATs at lower frequencies.

The high dispersion measure (DM, the integrated column density of free electrons along the line of sight, see Sect. 2.2 for more detail) of FRBs is caused by the contribution from the interstellar medium of our galaxy, the intergalactic medium, and the interstellar medium of the host galaxy. Each of the three regions could in principle be the main contribution of the high dispersion: for instance the intergalactic medium, an ionized medium around the source, or a cloud of ionized material in between the source and the observer. A cloud of ionized material has not been detected in the Milky Way for the first known FRB (Lorimer et al. 2007), but this explicit check is not performed/published for all FRBs (e.g., Thornton et al. 2013; Champion et al. 2016). An ionized medium around the source has been suggested for two FRBs: to explain either the scattering and Faraday rotation of FRB110523 (Masui et al. 2015) or the steep spectrum of FRB121102 (Kulkarni et al. 2015). In both cases, the bursts are still thought to be extragalactic, as was afterwards proven for FRB121102 (Tendulkar et al. 2017). If most of the DM contribution is because of the intergalactic medium, the FRBs are placed at redshifts of $0.2-1$ and must be intrinsically very bright (e.g., $10^{38}$ erg for FRB121102 at $z=0.19273(8)$; Tendulkar et al. 2017).

The initial single pulse nature observed for FRBs suggested a cataclysmic scenario; for example, evaporating black holes (Rees 1977), coalescing neutron stars (Hansen \& Lyutikov 2001), core-collapse supernova (Egorov \& Postnov 2009), and the collapse of a supra-massive rotating neutron star (or "Blitzar", Falcke \& Rezzolla 2014).

An exception to this scenario is the repeating FRB121102 (Spitler et al. 2014), where other models are required to explain the repetitions. These models include collisions between neutron stars and asteroids (Geng \& Huang 2015; Dai et al. 2016), soft gamma-ray repeaters (Popov \& Postnov 2007), magnetars (Popov \& Postnov 2013; Kulkarni et al. 2014), and signals enhanced by pulsar-orbiting bodies (Mottez \& Zarka 2014; a repeating model is in preparation). As repetitions have not been found for other FRBs, it is possible that cataclysmic and repeating FRBs form two different classes of objects.

The recurrence of FRB121102 made its localization possible at a high angular resolution (Chatterjee et al. 2017; Marcote et al. 2017). Optical observations identified the host to be a dwarf galaxy at a redshift of $z=0.19273(8)$, demonstrating its extragalactic origin (Tendulkar et al. 2017).

The first FRBs were detected at $1.4 \mathrm{GHz}$ by the Parkes Radio Telescope (Lorimer et al. 2007; Thornton et al. 2013; Champion et al. 2016), and later also by Arecibo (Spitler et al. 2014) and ASKAP (Bannister et al. 2017). Since then, searches have also been performed at lower frequencies with several detections at around $800 \mathrm{MHz}$ : one FRB by the GBT (Masui et al. 2015), three by the Molonglo telescope (Caleb et al. 2016a), and at least one by CHIME (Boyle \& CHIME/FRB Collaboration
2018). Searches at even lower frequencies using the GBT (Chawla et al. 2017, 350 MHz), Arecibo (Deneva et al. 2016, $327 \mathrm{MHz}$ ), LOFAR (Coenen et al. 2014; Karastergiou et al. 2015, $145 \mathrm{MHz}$ ) and the MWA (Tingay et al. 2015; Rowlinson et al. $2016,154+182 \mathrm{MHz}$ ) only set upper limits on the FRB rates.

Despite the high rates reported $\left(6_{-3}^{+4} \times 10^{3} \mathrm{FRBs} \mathrm{sky}^{-1}\right.$ day $^{-1}$; Champion et al. 2016), only about 40 FRB sources have been discovered so far (Petroff et al. 2016, FRBCAT ${ }^{1}$ ). This is mainly due to the limited field of view of the telescopes used. New techniques, such as phased array feeds (e.g., the Bannister et al. (2017) study that used ASKAP data) and aperture arrays, may increase the detection rate significantly.

FRBs have been observed in a range of pulse durations and DMs. Of the first 29 verified FRBs in FRBCAT, the shortest FRB has a width of $0.35 \mathrm{~ms}$. There are 4 FRBs with a width smaller than $1 \mathrm{~ms}, 15$ with a width of $1-4 \mathrm{~ms}, 6$ with a width of $4-8 \mathrm{~ms}$, and 4 with a width of more than $8 \mathrm{~ms}$. These widths are the measured duration and can be intrinsically narrower, but due to propagation and instrumental effects, can be smeared out over a longer period giving the observed duration. Instrumental effects are known to cause DM smearing due to the channelization of the data used by low-frequency surveys. The measured DMs for these 29 FRBs range from $176.4 \mathrm{pc} \mathrm{cm}^{-3}$ to $2596.1 \mathrm{pc} \mathrm{cm}^{-3}$, with 6 FRBs having a DM below $500 \mathrm{pc} \mathrm{cm}^{-3}$ and a further 3 in the $500-600 \mathrm{pc} \mathrm{cm}^{-3}$ range.

The localization of FRBs is important to help describe their origins but this information is often lacking. In the case of the repeating FRB, its localization was accomplished using sensitive single-dish telescopes and interferometers on a local or even global scale (Marcote et al. 2017). For non-repeating FRBs, localization is more difficult and has not yet been demonstrated. One possible way to achieve this is to have simultaneous interferometric and beamformed observations at similar frequencies. Non-simultaneous observations are also possible by triggering an interferometer working at a lower frequency than the detecting telescope. To this end, the LOw Frequency ARray (LOFAR; operating at $10-250 \mathrm{MHz}$; van Haarlem et al. 2013) is a telescope of interest as it is capable of both beamforming and interferometry, and has a wide field of view (11 square degrees per beam at $150 \mathrm{MHz}$ ), meaning that it can be used for both strategies. Here we present a real-time search strategy using LOFAR aimed at detecting and localizing FRBs.

The outline of this paper is as follows. Section 2 describes LOFAR and the FRATS project, Sect. 3 describes how limits are derived from the observations while Sect. 4 presents the results of the measurements, including upper limits on FRBs. This limit is compared with other measurements and predictions in Sect. 5. A conclusion is presented in Sect. 6.

\section{The FRATS project}

The aim of the Fast Radio Transient Search (FRATS) project is to search for individual outbursts of transient radio emission such as FRBs and RRATs and to localize them. An aperture array such as LOFAR can do this by combining time-domain methods to detect transients using a subset of the array and imaging techniques for localization with the entire array. FRATS consists of custom-written real-time triggering algorithms using streaming beamformed data, and the use of off-line imaging techniques

1 http://frbcat.org; we note that not all parameters, e.g., regarding scattering, are displayed by default, but they can be selected by clicking "visible columns". 
on the buffered data from individual tiles of the LOFAR stations to obtain a localization. The initial focus is on dispersed pulses of $2-128 \mathrm{~ms}$ in length and a fluence of the order of $1 \mathrm{kJy}$ ms.

A brief introduction to LOFAR, the real-time trigger, and the off-line imaging techniques is given below, followed by an introduction to the setup of pilot surveys. The details of the analysis pipelines are described in ter Veen (2015, hereafter TV15). This paper presents the results of the first three pilot surveys. Two surveys are used to set limits on the rate of FRBs at low frequencies and to give predictions for further observations. The third survey demonstrates the feasibility of commensal observing for the FRATS project.

\subsection{LOFAR}

LOFAR is a very flexible radio telescope as most of the signal processing is digital. LOFAR consists of stations separated by distances from less than $100 \mathrm{~m}$ to over $1000 \mathrm{~km}$. Each station consists of fields with 48-96 antennas that can be pointed in multiple directions at once. The signals from the different stations can be combined in different ways.

The specific features of LOFAR used for this project are the ability to create incoherent array beams with a large field of view, the ability to form these beams in parallel during other observing modes, and the ability to store the raw signal from each of the thousands of antennas in ring buffers known as transient buffer boards (TBBs). The ability to do commensal observing allows for a large duty cycle. The TBB data contain the last 5.2 (previously 1.3) seconds of the observation as raw voltages, which can be read out to study transient events in great detail. So far they have mainly been used for cosmic ray research (e.g., Schellart et al. 2013; Buitink et al. 2016), but this research uses them for sky localization of fast radio transients and detailed analysis of signals (TV15, Sect. 4). For the case of FRBs, localization is very important to identify host galaxies, and thus redshifts and energies (Tendulkar et al. 2017).

\subsection{The real-time triggering algorithm}

The FRATS real-time triggering algorithm consists of four steps: radio-frequency interference (RFI) mitigation, dedispersion into four or five sub-bands, pulse searching in these sub-bands, and a coincidence trigger across the sub-bands. It was designed to trigger with very low latency and to have a low false-detection rate. The trigger time is close to $3 \mathrm{~s}$ : that is, $1 \mathrm{~s}$ to obtain the data at the correlator, $1 \mathrm{~s}$ to beamform the data, and $1 \mathrm{~s}$ to analyze them in the FRATS pipeline. Occasionally, the process takes longer, for example due to data containing a lot of RFI. To make sure the process runs close to real-time, the data are analyzed in chunks of typically five-minute blocks. This ensures that, if this chunk is delayed, the next chunk may still run in real-time. In this way the raw data are still within the five-second TBB buffer.

All steps of the triggering algorithm are described in detail in TV 15 (Sects. 3.3 and 5.2). Here only a short description is given of the four steps:

(1) The RFI mitigation removes narrow-band interference by assuming the data are Gaussian in each channel and flagging the channels that deviate from that. It also flags wide-band local transient events that show no dispersion, but are visible simultaneously in at least two out of four sub-bands.

(2) The dedispersion algorithm corrects for the dispersive delay induced by free electrons on the arrival time of radio waves.
The number of free electrons is referred to as the DM. The $\mathrm{DM}$ is given by $\mathrm{DM}=\int n_{\mathrm{e}}(l) \mathrm{d} l$ with $n_{\mathrm{e}}$ the number density of free electrons along the path between the source and the observer. The delay $\Delta t$ between signals arriving at frequency $v_{1}$ and $v_{2}$, both in gigahertz, is calculated as

$\Delta t \approx 4.15 \mathrm{~ms} \mathrm{DM}\left(\frac{1}{v_{1}^{2}}-\frac{1}{v_{2}^{2}}\right)$.

The DM is different for each pulsar and transient burst, and therefore a search over this parameter is performed. Incoherent dedispersion is applied in five separate sub-bands of 6.25 MHz. The dispersive correction is applied at the integer sample level, for example, $2 \mathrm{~ms}$. The five sub-bands are used for three reasons: to provide a coincidence trigger (step 4) that significantly reduces the false trigger rate, for dedispersion efficiency as a wider band needs more DM trials, and to match the sensitivity of the TBB data analysis, which is limited by the bandwidth in the five-second buffer for the high-DM pulses.

(3) Each of these sub-bands is searched for a signal above a threshold, over different integration times, using a boxcar profile. The threshold is the average $+\mathrm{N} \sigma$, where $\sigma$ is the standard deviation in each sub-band (typically $N=7$ ). Outliers are not taken into account when calculating the average and standard deviation.

(4) Lastly, a coincidence algorithm checks that three out of five sub-bands trigger within a time window of $200 \mathrm{~ms}$, allowing for a delay between the sub-bands caused by the offset between trial DM and physical DM. In theory, a smaller window for this is possible. The coincidence trigger cuts the false trigger rate from over a thousand triggers per hour to a rate of one per hour. This is essential for a real-time trigger system that uses a TBB data read-out, as the read out takes from $30 \mathrm{~min}$ to $3 \mathrm{~h}$ and produces a data volume of up to $8 \mathrm{~TB}$ per event.

Because of the coincidence algorithm, the triggering is most sensitive to broadband pulses. This is the case in general, as narrowband pulses integrated over a larger frequency range will reduce the sensitivity. FRBs that show a particular narrowband structure can be missed if only one or two sub-bands are triggered. This effect is reduced by requiring that three instead of all five sub-bands are triggered, such that an on-off-on-off-on pulse will also trigger. Most FRBs show a broadband spectrum, but FRB 121102 is known to emit over a limited frequency range, which is different per pulse. At $3 \mathrm{GHz}$, this range is $290-690 \mathrm{MHz}$ (Law et al. 2017), thus much larger than the $6 \mathrm{MHz}$ bandwidth used in this survey. It is, however, not known what the behavior would be at low frequencies. If it scales as $\Delta v \sim$ $v^{4.4}$ like scintillation (Cordes et al. 1985), the bandwidth at $150 \mathrm{MHz}$ would be $\sim 1 \mathrm{kHz}$, much smaller than the $6 \mathrm{MHz}$ bandwidth, and thus not a problem for the coincidence algorithm.

\subsection{Off-line analysis and localization}

Upon triggering, the TBBs are frozen. The astronomer, alerted by e-mail, inspects the beamformed data by eye to verify that the trigger is from a real astrophysical source. If the data are interesting, the TBB is read out to disk for off-line analysis. For the full $5 \mathrm{~s}$ of data the read-out currently takes $3 \mathrm{~h}$. By using a parallel read-out, this may be reduced to $30 \mathrm{~min}$ in the future. The raw TBB data can be used to create pointings 
in any direction, though above $100 \mathrm{MHz}$ it is mostly sensitive within the tile beam and side lobes. A high-resolution image is created, using long-baseline stations to find its precise location. In principle, it is possible to obtain up to arcsecond resolution if good (ionospheric) calibration can be achieved when adding the international LOFAR stations. Given the long read-out time, only the first pulse of an RRAT or repeating FRB can be localized using the TBB data. However, reading out the TBB data does not stop the observation and subsequent pulses would still be detectable in the beamformed data, as long as the source is still being observed. The long duration of LOFAR observations ( $1 \mathrm{~h}$ for Pilot $\mathrm{A}$ and $\mathrm{B}$, and $8 \mathrm{~h}$ for a typical interferometric observation) gives a high probability of observing repeating pulses in a commensal survey.

\subsection{Analysis setup}

The first FRATS pilot surveys ran commensally during observations of the LOFAR tied array all-sky survey (LOTAAS) ${ }^{2}$. The three incoherent array beams already produced by the survey at $119-151 \mathrm{MHz}$ were used. Data were recorded for $1 \mathrm{~h}$ per pointing. The data were analyzed in 5 sub-bands of 512 channels of $12.207 \mathrm{kHz}$, then downsampled from $81.92 \mu \mathrm{s}$ to $491.52 \mu \mathrm{s}$ and further downsampled for the analysis to 2 or $4 \mathrm{~ms}$. Data were analyzed in blocks of 3-10 min, with the majority of blocks being $5 \mathrm{~min}$ in duration. There were two different setups, Pilot A and B, whose settings are displayed in Table 1. Pilot B used a DM range of $0-500 \mathrm{pc} \mathrm{cm}^{-3}$, while the upper DM of Pilot A was variable, depending on the available compute power, with 3,11 , 12 , and 10 observations using a DM range of 0 to $70,90,110$, or $120 \mathrm{pc} \mathrm{cm}^{-3}$, respectively. These ranges were chosen according to the maximum amount of data that could be stored in the TBBs (1.3 s at that time) that still allowed for enough bandwidth to localize sources. An upgrade of the TBBs to $5 \mathrm{~s}$ allowed the larger DM range to be searched. The DM step for pilot A was $0.3 \mathrm{pc} \mathrm{cm}^{-3}$ and the data were first downsampled to a $1.99608 \mathrm{~ms}$ time resolution. Pilot B used a DM step of $1.0 \mathrm{pc} \mathrm{cm}^{-3}$ with the data downsampled to $3.99216 \mathrm{~ms}$. The dedispersed timeseries were searched for pulses, using sliding windows between 2 and $16 \mathrm{~ms}$ for the first setup and between 4 and $128 \mathrm{~ms}$ for the second setup with power-of-two steps. Pilot A observed between 201301-16 and 2013-03-07 and Pilot B between 2013-08-09 and 2014-04-09.

In addition, Pilot $\mathrm{C}$ was completed with a different setup. Pilot $\mathrm{C}$ is presented to demonstrate the commensal observing capability during interferometric observations and is not used to derive limits on the rate of FRBs. An incoherent array beam was formed parallel to observations of the Radio Sky Monitor (Fender et al. 2008) project using all available stations. This is a 24-h zenith observation that searches for transients in the imaging domain on second to minute timescales, and on even longer timescales between epochs. Observations were taken on 201401-15 and 2014-01-16. It uses 11-min observations of six fields at declinations $+50.94^{\circ},+52.90^{\circ}$, and $+54.86^{\circ}$, each separated by a two-minute calibrator observation. Each field is observed twice. The observations are performed in four frequency bands of $2 \mathrm{MHz}$ width centered at $123.9,148.9,156$, and $184.9 \mathrm{MHz}$, respectively. The FRATS trigger ran on two parts of $5 \mathrm{~min}$ for each observation. The DM range covered was $0-500 \mathrm{pc} \mathrm{cm}^{-3}$, in steps of $1 \mathrm{pc} \mathrm{cm}^{-3}$, and the trigger threshold was $7 \sigma$. This is, to our knowledge, the first survey that used beamforming and imag-

\footnotetext{
2 http://www.astron.nl/lotaas/
}

Table 1. Observation and trigger properties.

\begin{tabular}{|c|c|c|}
\hline Property & Pilot A & Pilot B \\
\hline Incoherent array beams & 3 & \\
\hline Frequency range $(\mathrm{MHz})$ & $119-151$ & \\
\hline Pointing duration $(\mathrm{h})$ & 1 & \\
\hline Pointings & 35 & 83 \\
\hline Effective observing time (beam h) & 68.51 & 98.76 \\
\hline Frequency resolution $(\mathrm{kHz})$ & 12 & \\
\hline Time resolution (data) (ms) & 0.5 & \\
\hline Time resolution (search) (ms) & 2 & 4 \\
\hline Search width (ms) & $2,4,8,16$ & $4,8,16,32,64,128$ \\
\hline Station configuration & Superterp ${ }^{a}$ & \\
\hline FWHM 1 beam $119 \mathrm{MHz}$ (zenith) & $4.77^{\circ}$ & \\
\hline FWHM 1 beam $151 \mathrm{MHz}$ (zenith) & $3.78^{\circ}$ & \\
\hline FoV 1 beam $119 \mathrm{MHz}$ & $17.9 \mathrm{deg}^{2}$ & \\
\hline FoV 1 beam $151 \mathrm{MHz}$ & $11.25 \mathrm{deg}^{2}$ & \\
\hline Minimum DM $\left(\mathrm{pc} \mathrm{cm}^{-3}\right)$ & 0 & \\
\hline Minimum trigger $\mathrm{DM}\left(\mathrm{pc} \mathrm{cm}^{-3}\right)$ & 5 or 10 & 10 \\
\hline DM step $\left(\mathrm{pc} \mathrm{cm}^{-3}\right)$ & 0.3 & 1.0 \\
\hline Maximum DM $\left(\mathrm{pc} \mathrm{cm}^{-3}\right)$ & $70-120$ & 500 \\
\hline Sub-band bandwidth (MHz) & 6.25 & \\
\hline Number of sub-bands & 5 & \\
\hline Sub-bands required to trigger & 3 & \\
\hline Coincidence window (ms) & $2 \times 100$ & \\
\hline Single sub-band threshold (sigma) & 7 & \\
\hline
\end{tabular}

Notes. Pilot B values are only shown when different from Pilot A. (a) Dense core of 12 HBA substations.

ing techniques to search for transients of millisecond and minute duration, simultaneously.

\section{Event rate derivation}

In order to find a rate for the occurrence of transients, the sky rate $\mathcal{R}_{\text {sky }}$ at a certain fluence $\mathcal{F}$ is obtained by summing over each observation $o$ the minimum beam size $B$ across the band multiplied by the effective duration $D$ of a pointing, for all observations with a detectable fluence $\mathcal{F}(o)$ lower or equal to the threshold $\mathcal{F}$. The rate is the number of detections $N$ divided by this sum. Thus the expected rate in the case of $N$ detections is

$\mathcal{R}_{\text {sky }}(\mathcal{F})=N / \sum_{\mathcal{F}>=\mathcal{F}(o)} B(o) * D(o)$.

Similarly, the volume rate $\mathcal{R}_{\mathrm{vol}}$ is obtained by summing over the extragalactic volume $V$ that is searched in each observation

$\mathcal{R}_{\mathrm{vol}}(\mathcal{F})=N / \sum_{\mathcal{F}>=\mathcal{F}(o)} V(o) * D(o)$,

where $V$ is determined by the beam size and by the DM range searched, as this limits the redshifts searched (Sect. 3.4). The coverage is therefore a function of the beam size, volume (DM), duration, and sensitivity of each pointing. In this section the effective beam size, volume, duration, and sensitivity are discussed.

In case of non-detections $(N=0)$, one can use Eqs. (2) and (3) with $N=1$ to obtain an estimate for the upper limit for the sky and volume rates. Using Poissonian statistics, the obtained rate for $N=1$ then gives a chance of $1 / e \approx 36 \%$ to obtain $N=0$. We also adopt this common choice (Coenen et al. 2014; Karastergiou et al. 2015) in this paper. 


\subsection{Effective observing time and beam size}

The effective observing time is reduced due to the presence of RFI that is too strong to be removed by our cleaning process, and because of incomplete dedispersion at the start and end of a chunk of data. The latter is DM dependent. For Pilots A and B this reduces the observing time by $15 \%$ and $42 \%$, respectively, at the highest DM searched. The remaining observing time is referred to as the effective observing time and is used for setting limits.

The beam size of a LOFAR station depends on elevation (EL) as $1 / \sin (E L)$, in addition to the well-known dependency on frequency. The field of view (FoV) in Table 1 corresponds to the minimum FoV at an elevation of $90^{\circ}$ (zenith).

\subsection{Sensitivity}

To set a limit on the transient rate, the detection threshold needs to be determined. The noise level for an incoherent array beam of $N_{s}$ sub-stations at zenith is determined by $S_{\text {sys }}=$ SEFD $/ \sqrt{2 \Delta t \Delta v N_{s}}$, where SEFD is the system equivalent flux density, $\Delta t$ is the integration time, and $\Delta v$ is the bandwidth. For $\Delta t=2 \mathrm{~ms}$ and $\Delta v=6.25 \mathrm{MHz}$, the $S_{\text {sys }}=6.5 \mathrm{Jy}$ around $120 \mathrm{MHz}$ or $S_{\text {sys }}=5.5 \mathrm{Jy}$ from $130 \mathrm{MHz}$ to $150 \mathrm{MHz}$, (following van Haarlem et al. 2013). The value at $120 \mathrm{MHz}$ is used as the highest and therefore most conservative value when calculating $S_{\text {sys }}$. The effective bandwidth is decreased by $10 \%$ on average by flagged channels because of the two-step channelization ( 1 out of 16 channels, $6 \%$, contains no information because of the polyphase filter DC channel) and RFI (4\%). The noise level will also depend on the elevation EL. To correct for this, we use an antenna model with a simple correction factor of $\sin (E L)$. This is in agreement with more complex models (e.g., Hamaker 2011) within 5\%. In addition, the noise level is divided by 0.73 to compensate for unequal gain across the beam (following e.g., Edwards et al. 2001).

The noise level also depends on the sky temperature $T_{\text {sky }}$ which depends on the observing direction. The sky temperature towards the Galactic plane is higher, so an additional correction is applied to the $\mathrm{SEFD}$, as $\mathrm{SEFD}=\mathrm{SEFD}_{0}\left(T_{\text {sky }}+\right.$ $\left.T_{\text {inst }}\right) /\left(T_{0}+T_{\text {inst }}\right) . T_{\text {sky }}=T_{\text {Haslam }} * \lambda_{0}^{2.55}$, where $\lambda_{0}$ is the wavelength at $120 \mathrm{MHz}(2.5 \mathrm{~m}), \mathrm{T}_{\text {Haslam }}$ is the temperature from the $408 \mathrm{MHz}$ map by Haslam et al. (1982), corrected to a wavelength of $1 \mathrm{~m}$ using a spectral index of 2.55 (Lawson et al. 1987). $T_{0}=60 * \lambda^{2.55} \mathrm{~K}$ is the average temperature used to calculate $\mathrm{SEFD}_{0}$, and $T_{\text {inst }}=400 \mathrm{~K}$ is the instrumental noise as measured by Wijnholds \& van Cappellen (2011). An additional loss in sensitivity comes from the deviation of the real DM of the pulse from the trial DM used. For this situation, the overlap between the summed samples for the real DM and the trial DM can be calculated (TV15, Sect. 3.3.2). For Pilot A, the maximum loss is $25 \%$ for the $2-\mathrm{ms}$ pulses and $10 \%$ for 8 -ms pulses. For Pilot B, the maximum loss is $60 \%$ for $4-\mathrm{ms}$ pulses and $30 \%$ for $8-\mathrm{ms}$ pulses.

The DM smearing within one channel at these low frequencies is considerable. At a DM of $110 \mathrm{pc} \mathrm{cm}^{-3}$ (Pilot A) the smearing is $3.2-6.5 \mathrm{~ms}$ for $12 \mathrm{kHz}$ channels at 151 and $119 \mathrm{MHz}$, respectively. For Pilot $\mathrm{B}$, at a DM of $500 \mathrm{pc} \mathrm{cm}^{-3}$ the intrachannel smearing is $14.7-29.7 \mathrm{~ms}$ over the same range. When searching with a shorter sliding window, only part of the pulse is summed. For an $8-\mathrm{ms}$ pulse at a DM of $500 \mathrm{pc} \mathrm{cm}^{-3}$, the detection threshold therefore increases with a factor 3.7 or a loss of $73 \%$. However, this effect cancels out the loss in sensitivity caused by the deviation from the real DM, explained above, for shorter pulse lengths. The low DMs therefore have a threshold for Pilot B that is 2.6 times lower than that of the highest DM values. For Pilot A, this has no consequences for pulses of $8 \mathrm{~ms}$ and longer, even at the highest DMs, but shorter pulses will be smeared out.

The values presented above are for the design specification of LOFAR. This specification has probably not been reached, as there are likely additional noise contributions from improper calibration of the individual station beams, combining the individual beams into an incoherent array beam and additional system noise. Coenen (2013) compared the measured and expected signal-to-noise ratio $(\mathrm{S} / \mathrm{N})$ from 30 pulsars, and found that the gain was $40 \%$ of the predicted value. Therefore, in all our calculations, the noise level is increased by a factor 2.5 . This is a conservative value, as the Coenen (2013) measurements were done with an early installation of the system and are likely improved in the meantime by better calibration, and are less affected by additional noise because fewer stations are used for Pilots A and B in comparison with the Coenen (2013) measurements. Taking all this into account, the detection threshold for a pulse of $8 \mathrm{~ms}$ is of the order of $1 \mathrm{kJy} \mathrm{ms}$.

\subsection{Scattering}

Bhat et al. (2004) performed an empirical study on the effects of scattering on pulsar signals in the Milky Way. They found that pulse broadening scales with frequency and DM. The frequency scaling evolves as $\tau_{d} \approx v^{-\alpha_{1}}$ with $\alpha_{1} \sim 3.9 \pm 0.2$, giving 4000 times more scattering at $119 \mathrm{MHz}$ compared to $1 \mathrm{GHz}$. At $1 \mathrm{GHz}$, the scattering is on average $1 \mu \mathrm{s}$ at a DM of $50 \mathrm{pc} \mathrm{cm}^{-3}$, and $10 \mathrm{~ms}$ at a DM of $500 \mathrm{pc} \mathrm{cm}^{-3}$. There is a large spread in these values, so the scatter time can be a factor of ten higher or lower. Applied to $119 \mathrm{MHz}$, this yields scattering times of $4 \mathrm{~ms}$ and $40 \mathrm{~s}$ for a DM of $50 \mathrm{pc} \mathrm{cm}^{-3}$ and $500 \mathrm{pc} \mathrm{cm}^{-3}$, respectively. If this also holds for extragalactic pulses, the current search will not see any FRBs at the timescales probed. However, Lorimer et al. (2013) pointed out that, for extragalactic bursts, scattering is highest if a scattering screen is midway between the source and the observer. For FRBs, it is more likely that scattering originates from the host galaxy (less strong, because of redshifted emitting frequency) or our Galaxy, likely dependent on the observing direction. Therefore, the scattering may be expected closer to the value for a DM of $50 \mathrm{pc} \mathrm{cm}^{-3}$ in our Galaxy and at least some FRBs may be observed on millisecond timescales.

FRBCAT contains scattering times for half the FRBs ranging from 0.71 to $23 \mathrm{~ms}$, corrected to $1 \mathrm{GHz}$. These are low values compared to the Bhat et al. (2004) findings. However, if these are correctly measured and scale with frequency in the same way as pulsars, these pulses would be of the order of seconds at LOFAR frequencies, requiring longer integration time and thereby reducing the sensitivity of the measurements. Nonetheless, there may still be other FRBs with less scattering. More results by CHIME will be interesting for comparison in this regard. Their first detection (Boyle \& CHIME/FRB Collaboration 2018) shows no scattering on a pulse of $2 \mathrm{~ms}$ at a frequency as low as $580 \mathrm{MHz}$, and mentions other FRBs to be observable down to $400 \mathrm{MHz}$. For now, we assume there may still be FRBs at $8 \mathrm{~ms}$ at $119 \mathrm{MHz}$ and set limits accordingly; however, we also discuss the implications of scatter broadening to $128 \mathrm{~ms}$ where appropriate (Sects. 4.3.2, 5.1, and 5.6).

In general, we assume that scattering from intergalactic space can be neglected. It will not have an effect on the probed volumes for a given DM range. As long as we apply our limits only to the properties of observed pulses, we can disregard 


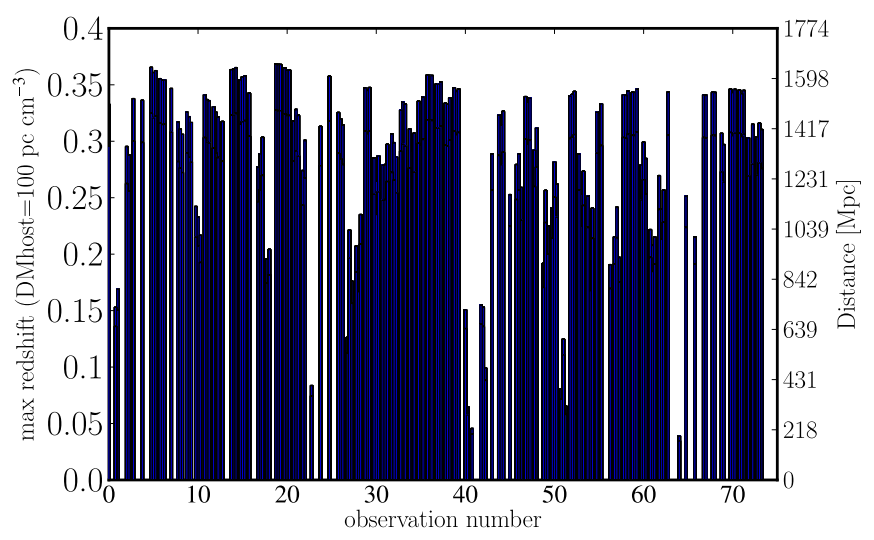

Fig. 1. Maximum redshift and intergalactic distance probed for each pointing of Pilot $\mathrm{B}$, assuming a $\mathrm{DM}_{\text {host }}=100 \mathrm{pc} \mathrm{cm}^{-3}$.

scattering in their derivation, and do so henceforth in this paper. However, due to the strong frequency dependence of scattering, scattering will have an effect when rates obtained at different frequencies are compared. If scattering is an erratic effect depending mainly on the line of sight, a fraction of the pulses may not be detected for a given experimental setup. This will essentially translate into a factor of less than one to be applied to the detection rate. We mention this whenever it affects our conclusions.

\subsection{Volume rate limit for extragalactic sources}

The DM in a certain direction depends on redshift $z$ as:

$$
\begin{aligned}
\operatorname{DM}(l, b, z) & =\operatorname{DM}_{\mathrm{MW}}(l, b)+\operatorname{DM}_{\mathrm{IGM}}(z)+\frac{\mathrm{DM}_{\text {host }}}{1+z} \\
& \approx \operatorname{DM}_{\mathrm{MW}}(l, b)+1100 z+\frac{100}{1+z}
\end{aligned}
$$

In these equations, $\mathrm{DM}_{\mathrm{MW}}$ is the predicted DM of the Milky Way at the Galactic coordinates $(l, b)$ of the observations using the NE2001 model (Cordes \& Lazio 2002), $\operatorname{DM}_{\mathrm{IGM}}(z) \approx 1100 z$ is the contribution from the intergalactic medium using Eq. (2) from Ioka (2003) in an approximation for $z<0.25$, and using the updated latest values for the constants of cosmology from Planck Collaboration XVI (2014). In this paper an estimated host DM of $100 \mathrm{pc} \mathrm{cm}^{-3}$ is used. Its contribution is corrected for the redshift of the transmitting frequency.

There are large uncertainties for both $\mathrm{DM}_{\mathrm{MW}}$ and for $\mathrm{DM}_{\text {host }}$. The value of $\mathrm{DM}_{\mathrm{MW}}$ cannot be measured in all directions and distances, and is therefore an interpolation and extrapolation of the points where it can be measured. The value of $\mathrm{DM}_{\text {host }}$ depends on galaxy type, inclination angle and can be increased by ionized clouds along the path. The only $\mathrm{DM}_{\text {host }}$ measured is for the repeating FRB121102 at DM $\leq$ $324 \mathrm{pc} \mathrm{cm}^{-3}$ (Tendulkar et al. 2017), which appears high compared to average DMs of $37 \mathrm{pc} \mathrm{cm}^{-3}$ for elliptical galaxies and $45 \mathrm{pc} \mathrm{cm}^{-3}$ for spiral galaxies (Xu \& Han 2015), although averaging over inclination increases the latter to $142 \mathrm{pc} \mathrm{cm}^{-3}$. This high DM can be caused by ionized material close to the source, because of a star forming region there (Bassa et al. 2017). A very high $\mathrm{DM}_{\text {host }}$ will decrease the $\mathrm{DM}_{\mathrm{IGM}}$ contribution significantly, and will therefore decrease the galactic volume searched. As whether or not this really is the typical DM for FRBs is unknown, because FRBs with a DM as low as $174 \mathrm{pc} \mathrm{cm}^{-3}$ have also been measured, we assume in our analysis a $\mathrm{DM}_{\text {host }}$ of $100 \mathrm{pc} \mathrm{cm}^{-3}$, after Lorimer et al. (2007), but also in line with

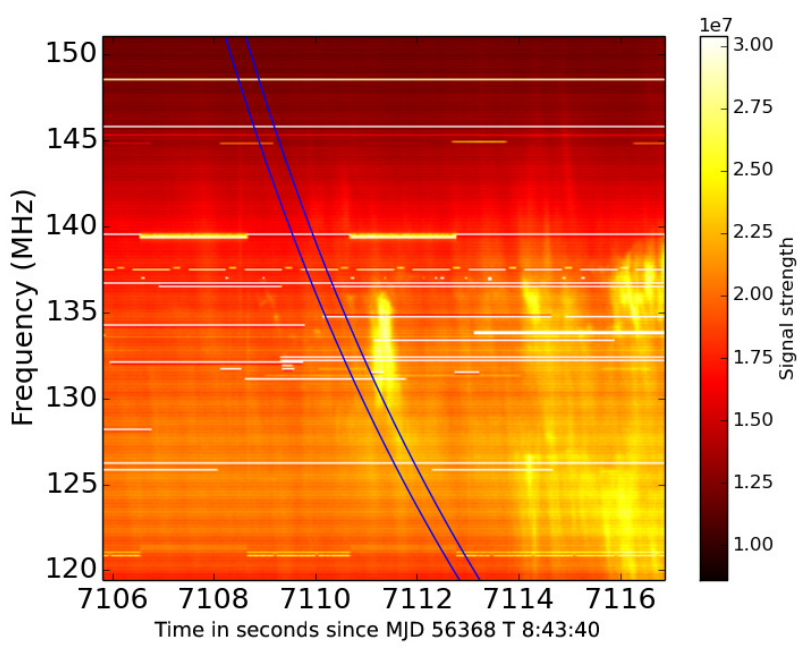

Fig. 2. Detection plot automatically created by the real-time algorithm. The signal is consistent with a type II solar radio burst. The blue lines denote the dedispersion relation around the trigger time. The plot uses arbitrary units.

some orientations of elliptical and spiral galaxies and an additional contribution from the local environment.

Equation (4) was used to give the maximum redshift corresponding to the maximum DM that was searched. The result is plotted in Fig. 1 for Pilot B. The CosmoloPy package ${ }^{3}$, following Hogg (1999), is used to find the co-moving distance, luminosity distance, and observed volume for that redshift. The co-moving distance is typically $\sim 1 \mathrm{Gpc}$, as shown in Fig. 1. With this method, the DM-limited volume for each observation is calculated as used in Eq. (3).

\section{Results}

Here we show results from the pilot surveys A, B, and C. From the first two we present the first exploratory results of the dispersed pulse search (Sects. 4.3.1 and 4.3.2). Pilot $C$ shows the potential of commensal observing during imaging observations (Sect. 4.2). We first show the detection of a solar radio burst as a proof of concept of the potential for localization with the TBBs.

\subsection{Localization of a solar radio burst}

Localization of FRBs is one of the most important goals for current developments in FRB searches. As a proof of concept of the potential for localization with the TBBs, we describe here the detection of a solar radio burst.

During observations of Pilot A, the real-time trigger detected peculiar emission at a DM of $43 \mathrm{pc} \mathrm{cm}^{-3}$. Figure 2 shows the original detection plot generated by the trigger software. The triggering emission is between the two blue lines that denote the dispersion relation. We note that we plot the time of the first trigger, instead of the time with the highest peak. The trigger occurred due to the highly non-Guassian behavior of the dedispersed sub-bands created by the emission from the solar radio burst, in combination with the normalization functions. Even though it is clear that this signal is not an FRB or pulsar, it is also not clear that it is regular RFI. Therefore, we recorded TBB data for further analysis.

roban.github.io/CosmoloPy/ 


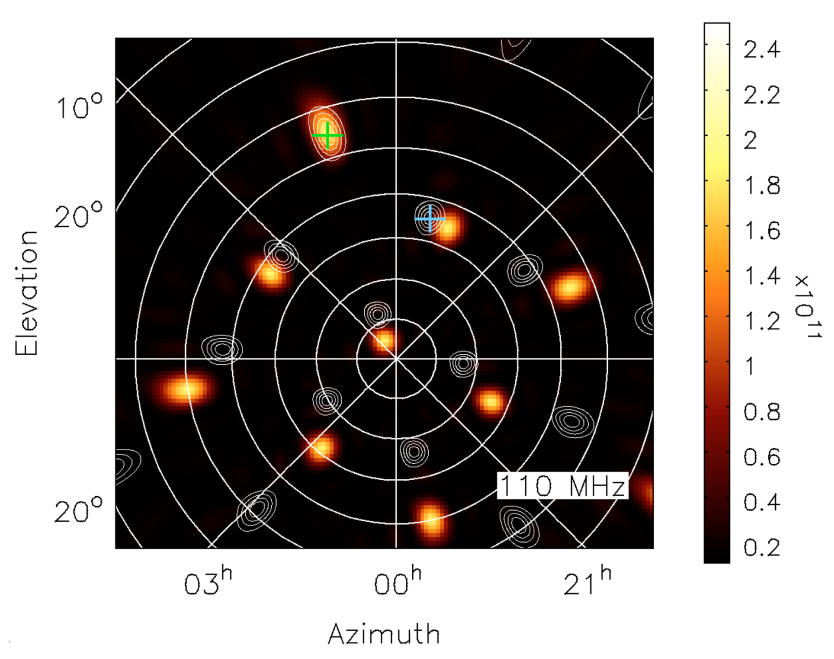

Fig. 3. All sky image from TBB data of a single LOFAR station showing the Sun (green cross) and the grating lobes (strong side lobe response cause by the regular antenna layout of a single station). The original observation was made in the direction marked by the blue cross. The color image is at $110 \mathrm{MHz}$, and contours are at $135 \mathrm{MHz}$. North is at $0^{\circ}$ Azimuth, and east to the left. The plot uses arbitrary units.

TBB data of one station (CSO03) was used to make allsky images at different frequencies. Figure 3 shows the all-sky images at 110 and $135 \mathrm{MHz}$ in color scale and contour lines, respectively. The different regularly spaced side lobes created by the antenna layout of a single station are clearly visible. The original direction of the LOFAR observation is shown with a blue cross at $13 \mathrm{~h}$ in azimuth, where 0 azimuth is north, and $6 \mathrm{~h}$ is east. This is a side-lobe detection, as the actual source is visible at both frequencies at $11 \mathrm{~h}$ (green cross). The source location corresponds to the position of the Sun at the time of the observation.The morphology of this emission is consistent with a type II radio burst (i.e., Cunha-Silva et al. 2015).

This example of a solar radio burst clearly shows the ability of the TBB data to find the event sky location, even if it is not coming from within the primary beam, and of the possibility to study nondispersed signals.

\subsection{Pulsar rediscoveries}

The main objective of the FRATS project is to find dispersed pulses. An obvious candidate source class is pulsars. All bright pulsars with an estimated peak flux density of more than $15 \mathrm{Jy}$ in the sky covered by the observations were detected, either online or in a later offline analysis (TV15, Sects. 3.4.1 and 5.3.1). Three pulsars were detected in Pilot A (PSRs B0834+06, B1133+16 and B1541+09) and seven in Pilot B (PSRs B2016+29, $\mathrm{B} 2111+46, \mathrm{~B} 0320+39, \mathrm{~B} 1919+21, \mathrm{~B} 2217+47, \mathrm{~B} 2021+51$ and $\mathrm{B} 0301+19)$. The pulsar with the highest DM detected was PSR B2111+46 with a DM of $141.26 \mathrm{pc} \mathrm{cm}^{-3}$, showing the potential for discovering FRBs.

Pilot B showed two interesting rediscoveries: PSRs B2021+51 and B0301+19. Based on flux densities from higher frequencies, they have an extrapolated peak flux density of only 11 and $2 \mathrm{Jy}$, respectively, while the trigger threshold is $100 \mathrm{Jy}$ for an 8-ms pulse. This indicates a broad distribution in pulse flux densities, a known behavior for these pulsars. In fact, these pulsars were originally detected in single pulse surveys at $408 \mathrm{MHz}$, where B0301+19 was generally weak, but occasionally emitted two or three bright pulses (Bonsignori-Facondi et al.
1973), and B2021+51 showed a large distribution in pulse amplitudes (Davies \& Large 1970). This behavior is still present at $135 \mathrm{MHz}$.

During the commensal Pilot $\mathrm{C}$ survey, five pulsars were rediscovered: PSR B0329+54, PSR B1120+50, PSR B1508+55, PSR B1953+50, and PSR B2021+51. They are shown in Fig. 4, where the right ascension, declination and DM of the triggers are compared with those of known pulsars in the field up to a DM of $100 \mathrm{pc} \mathrm{cm}^{-3}$. Most notable is PSR B1112+50, that has a weak average flux density, but is known to emit giant pulses (Ershov \& Kuzmin 2003; Karuppusamy et al. 2011). The successful rediscovery of five pulsars shows that LOFAR can run these two observing modes in parallel and detect dispersed pulses.

\subsection{Fast radio burst limits}

All dispersed pulses found can be attributed to known pulsars. From this, an upper limit on the occurrence of FRBs and other fast radio transients at $135 \mathrm{MHz}$ is derived in this section.

\subsubsection{Sky rate limit from Pilot $A$}

The rate limit in events per sky per day as a function of fluence is determined as described in Sect. 3. The result for Pilot A, based on an effective observing time of 68.5 beam $\mathrm{h}$, is presented in Fig. 5. This figure shows the observed sky for fluence thresholds between 1 and $2 \mathrm{kJy} \mathrm{ms}$, as calculated by Eq. (2). This is not the complete range that could be observed, but gives the best overview. Of the pointings, $40 \%$ have a fluence between 0.7 and $1 \mathrm{kJy} \mathrm{ms}$, while $10 \%$ have a fluence above $1.6 \mathrm{kJy} \mathrm{ms}$, and up to $6 \mathrm{kJy} \mathrm{ms}$ for pointings in the Galactic plane. Taking $90 \%$ of the pointings into account, we set a limit for the event rate of 1500 events per sky per day above a threshold of $1.6 \mathrm{kJy} \mathrm{ms}$ for an 8 -ms pulse. The pulse duration is important in this limit. For pulses shorter than $8 \mathrm{~ms}$, the average brightness needs to be larger, because of the intra-channel DM smearing and the decrease in efficiency caused by the finite DM step (see TV15, Sect. 5.2.1). For longer pulses, the minimum detectable fluence increases with $\sqrt{\Delta t}$, where $\Delta t$ is the pulse duration $\left(S_{\text {sys }}\right.$ goes down with $1 / \sqrt{\Delta t}$, but for fluence it is multiplied by the duration $\Delta t$ ). No volume rate is derived for Pilot $\mathrm{A}$, because the volume probed is minimal due to the limited DM range searched.

\subsubsection{Sky rate and volume rate limits from Pilot $B$}

In Pilot $\mathrm{B}$, triggers below a DM of $10 \mathrm{pc} \mathrm{cm}^{-3}$ were omitted because of the high probability that they are caused by local RFI. After the data-quality cut, including the DM-dependent data loss because of incomplete dedispersion at the start and end of a chunk, 91.2 beam $\mathrm{h}$ remained at a DM of $500 \mathrm{pc} \mathrm{cm}^{-3}$. A sky rate limit and a volume rate limit on isolated dispersed pulses have been calculated as a function of fluence. These are shown in Fig. 6 for an $8 \mathrm{~ms}, 32 \mathrm{~ms}$, and $128 \mathrm{~ms}$ pulse duration. Because of the DM smearing affecting the 8-ms results, the 32-ms results are $40 \%$ more sensitive, while the $128-\mathrm{ms}$ results are only $13 \%$ less sensitive than the 8 -ms results. The limit is 1400 events per sky per day, or equivalently 134 events per $\mathrm{Gpc}^{3}$ per day above a fluence of $6.0 \mathrm{kJy} \mathrm{ms}$ for an $8-\mathrm{ms}$ pulse up to a DM of $500 \mathrm{pc} \mathrm{cm}^{-3}$. Limits at a lower fluence threshold, for a smaller DM range, and for longer pulse durations are presented in Table 2. 

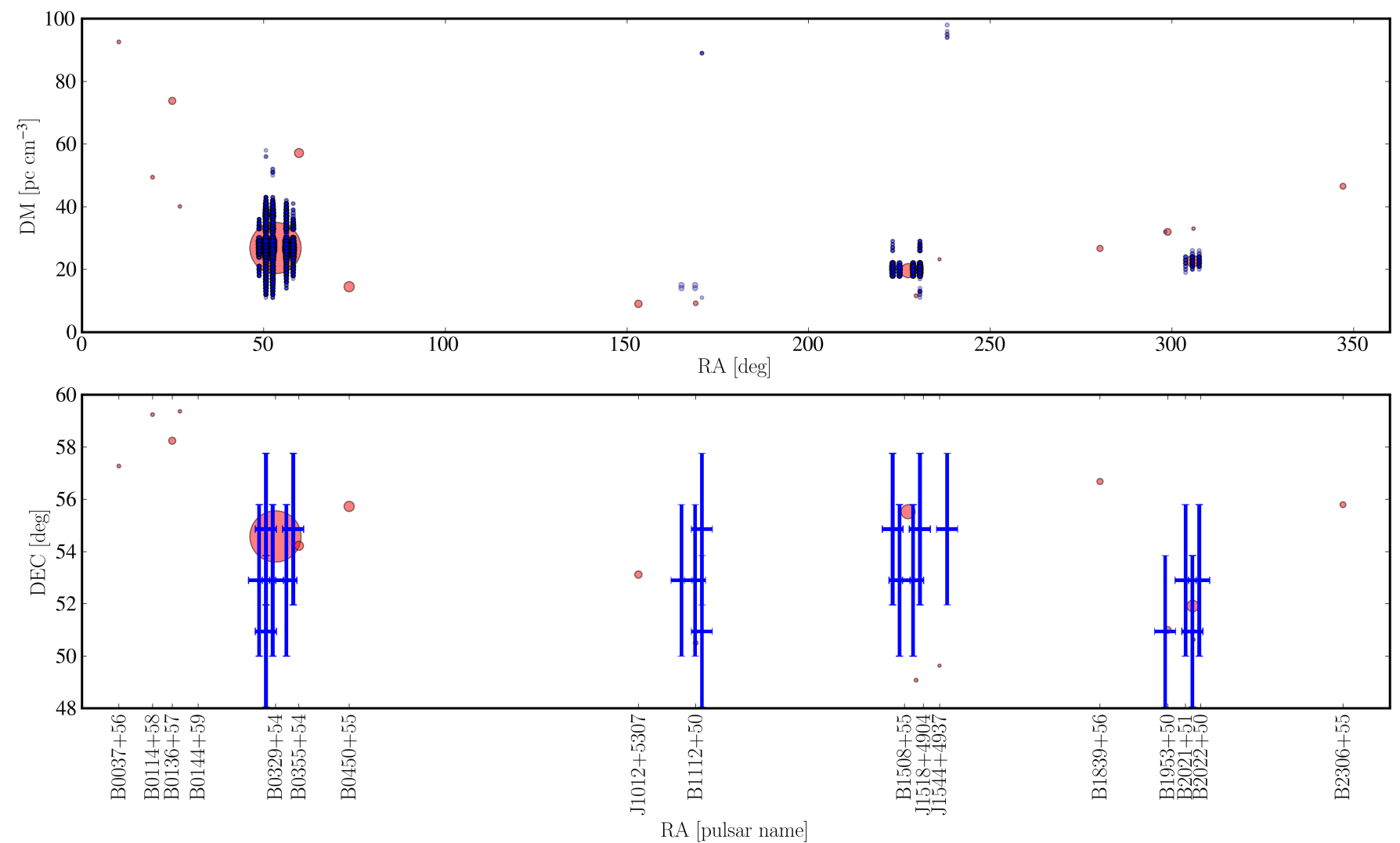

Fig. 4. Pulsars detected in Pilot C. Top plot: DM vs. RA for the known bright pulsars in red circles. The flux density at $400 \mathrm{MHz}$ is depicted by the sizes of the circles. Only pulsars with a flux density above $5 \mathrm{mJy}$ are shown. The smaller blue circles in the top plot denote the coincidence triggers. Bottom plot: pulsars and pointings that trigger are plotted now in Dec vs. RA. The error bars of the blue points denote the beam size. Pulsar names are plotted at the bottom. Circle scalings are linear.

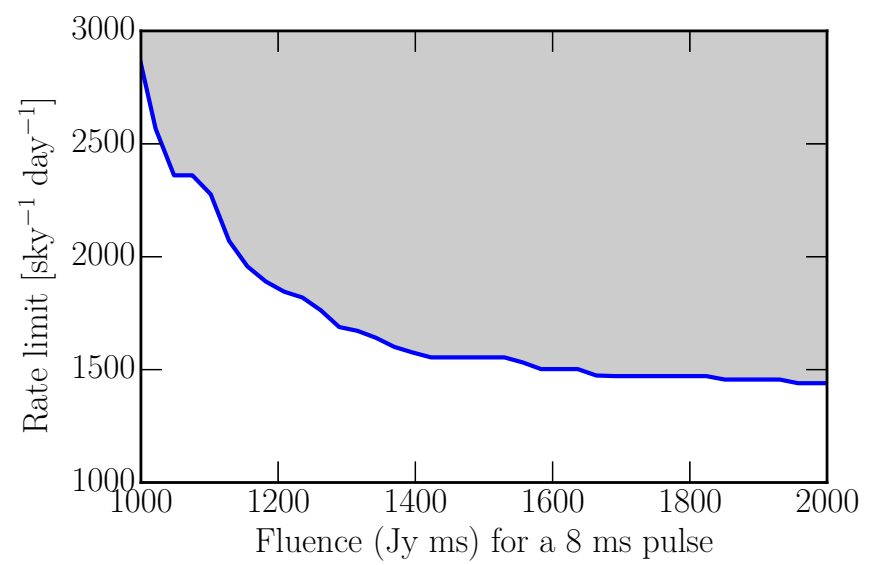

Fig. 5. The gray region indicates the parameter space excluded by a nondetection in Pilot A. The fluence is displayed from 1 to $2 \mathrm{kJy} \mathrm{ms}$, while the minimum and maximum trigger thresholds are at fluences of $0.7 \mathrm{kJy} \mathrm{ms}$ and $6 \mathrm{kJy} \mathrm{ms}$, respectively.

\section{Discussion}

In this section we compare the event rates from our nondetections to values in the literature. We first discuss the sky rate limits (Sect. 5.1) and the implications for RRATs (Sect. 5.2). Subsequently, we compare the rate per volume from Pilot B with expectations from Sect. 5.3 onwards, and discuss the options of a star formation history dependence (Sect. 5.4) and a nonuniform distribution (Sect. 5.5). Finally, we give an outlook on the detectability of FRBs with LOFAR (Sect. 5.6).

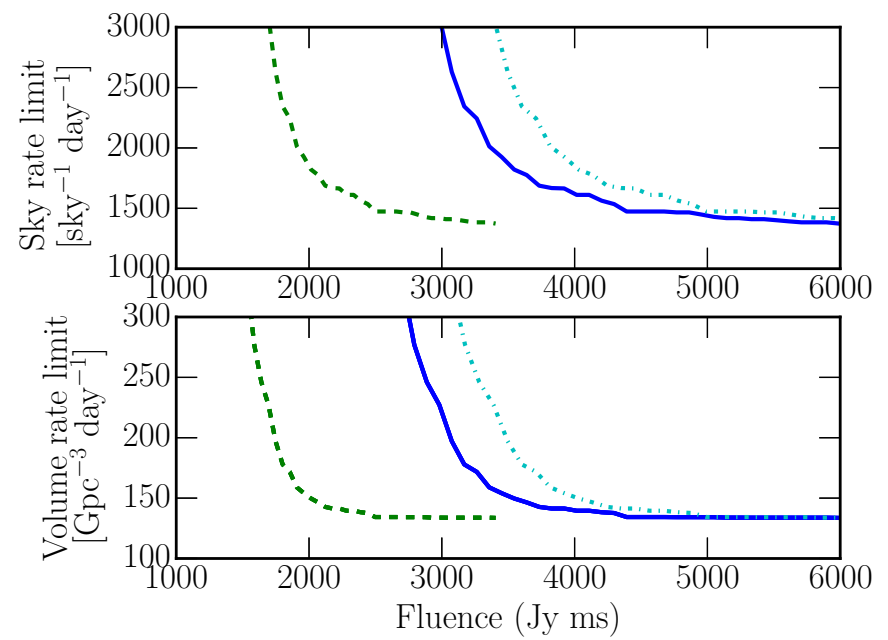

Fig. 6. Rate excluded by sky area covered (top plot) and volume covered (bottom plot) for Pilot B, as a function of pulse fluence, for three different integration lengths: $8 \mathrm{~ms}$ (blue line), $32 \mathrm{~ms}$ (green dashed line), and $128 \mathrm{~ms}$ (cyan dash-dotted line).

\subsection{Sky rate}

In Fig. 7, we compare the sky rate and upper limits from various surveys that were performed below $1 \mathrm{GHz}$, where fewer detections are available with respect to $1.4 \mathrm{GHz}$. The surveys considered $^{4}$ are summarized in Table 3. The MWA surveys were named

4 We note that the current fast pace of this field makes it likely that the list may be incomplete by the time of publication. 
Table 2. Rate limits for Pilots A and B for different minimum detectable fluence, observing lengths, and DM ranges.

\begin{tabular}{llrcc}
\hline \hline Survey & Rate limit & $\begin{array}{r}\text { DM range } \\
(\mathrm{pc} \mathrm{cm}\end{array}$ & $\begin{array}{c}\text { Min. Fluence } \\
(\mathrm{kJy} \mathrm{ms})\end{array}$ & $\begin{array}{c}\text { Length } \\
(\mathrm{ms})\end{array}$ \\
\hline Pilot A & $1500 \mathrm{sky}^{-1} \mathrm{day}^{-1}$ & $0-110$ & 1.6 & 8 \\
Pilot B & $1400 \mathrm{sky}^{-1} \mathrm{day}^{-1}$ & $10-135$ & 2.2 & 8 \\
Pilot B & $1400 \mathrm{sky}^{-1} \mathrm{day}^{-1}$ & $10-500$ & 6.0 & 8 \\
Pilot B & $2240 \mathrm{sky}^{-1} \mathrm{day}^{-1}$ & $10-500$ & 3.3 & 8 \\
Pilot B & $1400 \mathrm{sky}^{-1} \mathrm{day}^{-1}$ & $10-500$ & 3.4 & 32 \\
Pilot B & $1400 \mathrm{sky}^{-1} \mathrm{day}^{-1}$ & $10-500$ & 6.8 & 128 \\
Pilot B & $134 \mathrm{Gpc}^{-3} \mathrm{day}^{-1}$ & $10-500$ & 6.0 & 8 \\
Pilot B & $185 \mathrm{Gpc}^{-3} \mathrm{day}^{-1}$ & $10-500$ & 3.3 & 8 \\
\hline
\end{tabular}

Notes. Values for this table for Pilot B were taken as examples from Fig. 6.

by us. The results in this and subsequent sections are corrected for a pulse duration of $8 \mathrm{~ms}$, as most detected FRBs so far have a length shorter than this. Because of scattering, the pulse length may also be longer for lower frequencies leading to different detection thresholds. If the pulse length is of the order of $128 \mathrm{~ms}$ at $135 \mathrm{MHz}$, the sensitivity for FRATS is $13 \%$ less, and the previous surveys below $200 \mathrm{MHz}$ will lose about a factor of four in sensitivity, while the previous surveys above $320 \mathrm{MHz}$ are not affected as the scattering would be below $8 \mathrm{~ms}$. Our results for Pilot B are presented by the black line and shaded region in the upper right corner. Limits and results from other surveys are represented by the different shapes in the plot. The limit on the FRB rate determined by our results is comparable with the rate limit determined by the GBNCC survey, the GBTIM survey (Connor et al. 2016), and the MWA-154 survey, albeit at a different fluence threshold. In sensitivity, our results are comparable with AO327 and the MWA-154 survey. The only two published detections have been made by the GBTIM and UTMOST survey (Caleb et al. 2017). The latter reports a detectable rate limit of $0.78_{-0.57}^{+1.24} \times 10^{2}$ events per sky per day at a fluence above $11 \mathrm{Jy} \mathrm{ms}$ and a fluence complete rate limit of $5.0_{-4.7}^{+18.7}$ events per sky per day above a fluence of $69 \mathrm{Jy} \mathrm{ms}$.

The rate at different fluence thresholds and observing frequencies depends on how the number of FRBs changes as function of fluence, and how the fluence changes as a function of observing frequency. This is expressed by the relationships $\mathcal{R}\left(>\mathcal{F}_{\lim }\right) \propto \mathcal{F}_{\lim }^{\alpha}$ and $S \propto v^{\gamma}$, where $\mathcal{R}$ is the rate, $\mathcal{F}$ is the fluence, $\mathrm{S}$ the spectral energy distribution and $v$ the observing frequency. Caleb et al. (2017) find that if $\alpha=-3 / 2$, as in Euclidean space, the spectral index $\gamma=-1.1(1.2)$. If, on the other hand, $\gamma$ $=0$, they find $\alpha=-1.0(1.1)$. This is more in line with the evolution of volume with redshift; see Sect. 5.3. These relations are represented by the lines in Fig. 7, where the case of $\gamma=-1.1$ has been corrected to the survey frequency at $140 \mathrm{MHz}$. Given these rates, it is not surprising that our pilot surveys and the other surveys have not discovered FRBs so far.

The results from two other LOFAR surveys are also shown: the off-line survey LPPS (Coenen et al. 2014) and the real-time survey ARTEMIS (Karastergiou et al. 2015), both observing only one band of 7 and $6 \mathrm{MHz}$, respectively, around $145 \mathrm{MHz}$. They have reached a lower rate limit than this work because they use more beams and more observing hours than in our pilot surveys. They are also more sensitive due to the increased number of stations (LPPS) or better dedispersion accuracy (LPPS and ARTEMIS). ARTEMIS uses one international station that is $15 \%$ more sensitive than the LOFAR superterp combined incoherently. In addition, we applied a correction factor of 2.5 between the theoretical and actual sensitivity (Sect. 3.2), as was done for the LPPS survey. To the LPPS flux density threshold we apply an additional factor two to account for intra-channel DM smearing of LPPS at a DM of $500 \mathrm{pc} \mathrm{cm}^{-3}$ for an $8-\mathrm{ms}$ pulse. While LPPS analyzed the data up to a DM of $2000 \mathrm{pc} \mathrm{cm}^{-3}$, ARTEMIS limited itself to $320 \mathrm{pc} \mathrm{cm}^{-3}$, observing a smaller instantaneous volume than FRATS with a cut-off at $500 \mathrm{pc} \mathrm{cm}^{-3}$. Using all stations, instead of the 12 sub-stations used in the pilot survey, FRATS will be more sensitive than LPPS and ARTEMIS (see also Sect. 5.6). Also, unlike FRATS, they are not designed to use TBB data to localize pulses, which is the main goal of the FRATS project.

\subsection{Rotating radio transients}

RRATs are pulsars of which only single pulses are detected (Burke-Spolaor et al. 2013). The time between these pulses can last from minutes to hours. The long integration times of commensal observations gives FRATS a high potential of discovering RRATs with a long "off" time. Burke-Spolaor et al. (2011) detected RRATs with an off time of three times the observing time, which would extend this time even further. Pilot $A$ is more sensitive to RRATs, as they are local, meaning that the DM limit of $110 \mathrm{pc} \mathrm{cm}^{-3}$ is not a problem, but the DM search is more sensitive because of the smaller DM step. We determine that in Pilot A, covering 1610 square degrees (3.7\% sky), no bright RRATs were observed in a typical effective observation duration of $42 \mathrm{~min}$ (after data-quality cuts), above a threshold of $1.6 \mathrm{kJy} \mathrm{ms}$ for an $8-\mathrm{ms}$ pulse. Based on the RRATs population published (early) in the literature this is not surprising. Only 1 of approximately 60 RRATs (McLaughlin et al. 2006; Deneva et al. 2009; Burke-Spolaor \& Bailes 2010; Burke-Spolaor et al. 2011; Keane et al. 2011) would be bright enough to be detected by our observations, assuming RRATs have a pulsar-like average spectral index of -1.8 (Maron et al. 2000; Miller et al. 2011). Almost all RRATs require a sensitivity at least four times higher than that of our observations presented here to be detected. This is without taking scattering into account, which would further weaken the emission at low frequencies. With long commensal observations, in conjunction with additional stations to reach the sensitivity required, it would be possible to detect RRATs with off times of several hours.

\subsection{Volume rate, comparison, and expectations}

Because of intra-channel DM smearing, the low frequencies are restricted in their DM range and thus in the redshift range in which sources will be detected. Therefore, to compare survey results and make predictions, it is useful to compare the volume rate $\mathcal{R}_{\mathrm{vol}}$ (events per $\mathrm{Gpc}^{3}$ per day) rather than the sky rate $\mathcal{R}_{\text {sky }}$ (events per sky per day).

Hassall et al. (2013) determined $\mathcal{R}_{\mathrm{vol}}$ by simulations. Based on this study, we use an adjusted $\mathcal{R}_{\mathrm{vol}}$ of 12 events per $\mathrm{Gpc}^{3}$ per day (under the assumption that there is no scattering), by choosing the rate for the results by Thornton et al. (2013) as most reliable, and correcting it for the new $\mathcal{R}_{\text {sky }}$ of Champion et al. (2016).

The rate of 12 events per $\mathrm{Gpc}^{3}$ per day is still more than one order of magnitude less than the upper limit derived in this pilot survey, which is $\mathcal{R}_{\mathrm{vol}}<134$ events per $\mathrm{Gpc}^{3}$ per day. If these predictions are correct, it is clear that at least ten times more observing time is needed in order to be able to detect a similar event rate, even if all FRBs could be detected by our setup. A more recent result (Law et al.2017) predicts a ten times higher volumetric rate, based 


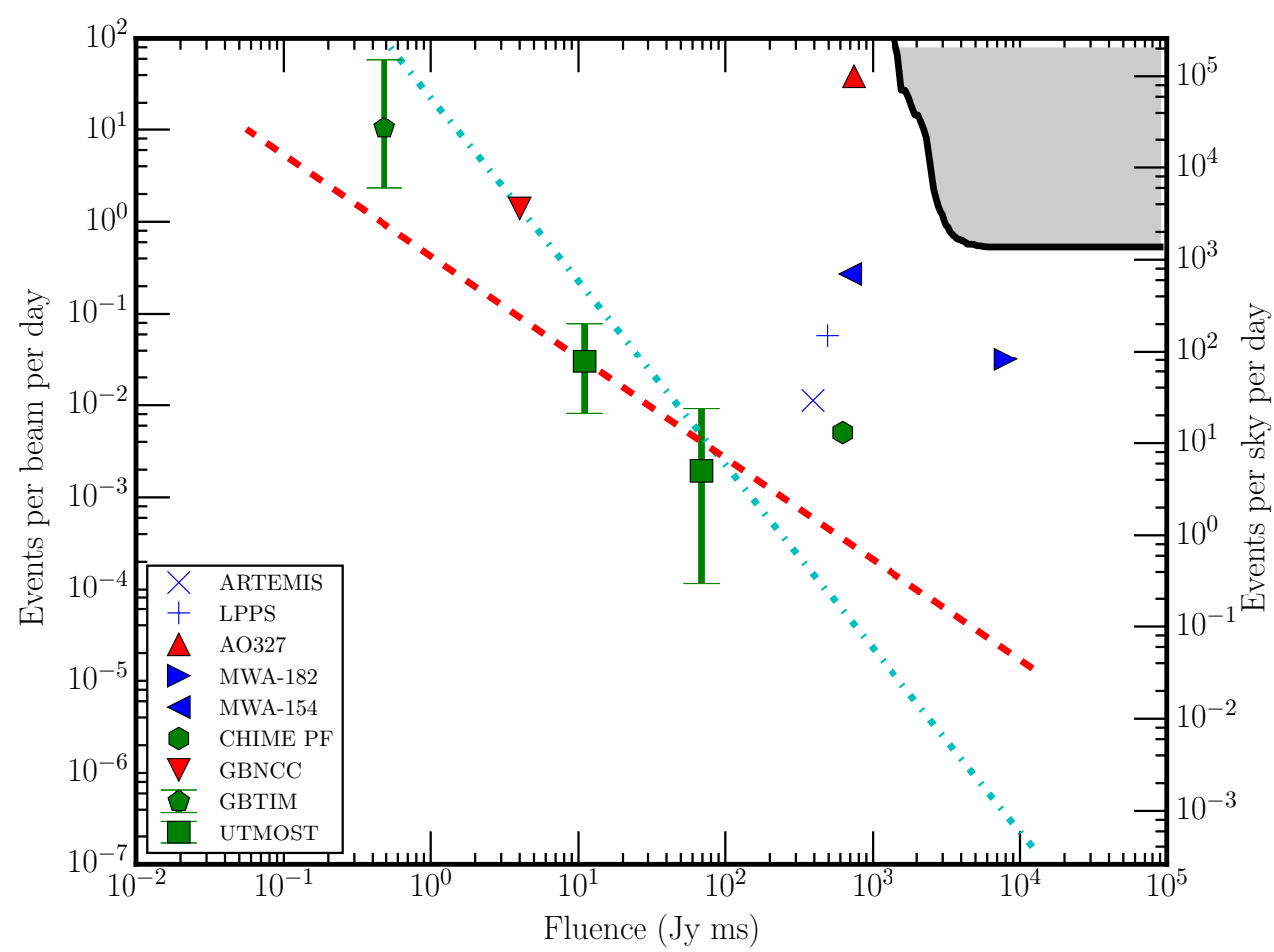

Fig. 7. Event rate vs. fluence for different surveys, corrected for a pulse length of $8 \mathrm{~ms}$. The limits from the results of Pilot B are presented by the line in the upper-right corner. The symbols represent rate (pentagram and squares) and upper limits for other instruments observing below $1 \mathrm{GHz}$, where green symbols observe around $800 \mathrm{MHz}$, red symbols around $350 \mathrm{MHz}$, and blue symbols below $200 \mathrm{MHz}$. Survey or telescope name, mid-frequency, DM range, and references are given in Table 3. The dashed red line assumes $\log ($ event rate $) / \log ($ fluence $)=-1$ and the cyan dash-dotted line assumes $\log$ (event rate) $/ \log$ (fluence) $=-3 / 2$, with an adjustment of the fluence assuming a spectral index of -1.1 to a reference frequency of $140 \mathrm{MHz}$
Table 3. Survey parameters for Figs. 7 and 9.

\begin{tabular}{llrlr}
\hline \hline & Survey & $\begin{array}{r}\text { Central Freq } \\
(\mathrm{MHz})\end{array}$ & $\begin{array}{l}\text { DM range } \\
\left(\mathrm{pc} \mathrm{cm}^{-3}\right)\end{array}$ & Reference \\
\hline$\square$ & GBTIM & 800 & $20-2000$ & 1 \\
$\nabla$ & GBNCC & 350 & $0-500$ & 2 \\
$\square$ & UTMOST & 843 & $100-2000$ & 3 \\
$\square$ & MWA-182 & 182 & $<0-700$ & 4 \\
$\triangleleft$ & MWA-154 & 154 & $<170-675$ & 5 \\
$\square$ & CHIME pf & 600 & $20-2000$ & 6 \\
$\Delta$ & AO327 & 327 & $0-1095$ & 7 \\
+ & LPPS & 142 & $0-2000$ & 8 \\
$\times$ & ARTEMIS & 145 & $0-320$ & 9 \\
-- & FRATS & 135 & $10-500$ & 10 \\
\hline
\end{tabular}

References. (1) Connor et al. (2016); (2) Chawla et al. (2017); (3) Caleb et al. (2017); (4) Rowlinson et al. (2016); (5) Tingay et al. (2015); (6) Amiri et al. (2017); (7) Deneva et al. (2016); (8) Coenen et al. (2014); (9) Karastergiou et al. (2015); (10) Pilot B.

on the assumption that the repeater FRB121102 is a prototype for the class, with a power of $10^{40}$ erg at $z=1$.

Another aspect required to detect FRBs is the brightness of the FRBs compared to the sensitivity of the instrument. To estimate this, we show in the upper panel of Fig. 8 the fluence of the first 20 known FRBs as a function of redshift. The fluence threshold of Pilot B is plotted as the horizontal dashed line for a flat spectrum at $\gamma=0$. From this plot, it is clear that all FRBs detected so far have a fluence, at their observed frequencies, below the threshold of Pilot B. There are two ways in which FRATS would still be sensitive enough to these FRBs. Firstly, an FRB that is closer appears brighter. Secondly, they could be intrinsically brighter at lower frequencies (spectral index $\gamma<0$ ). For the first option, we have extrapolated the fluence to lower redshifts for three cases: the brightest FRB (green line), the dimmest FRB ( blue line), and the median value (red line). From
Fig. 8 we see that even the brightest FRB detected so far would only be visible for $\gamma=0$ up to a redshift of 0.07 , corresponding to a DM of $\sim 210 \mathrm{pc} \mathrm{cm}^{-3}$. For the second option we have also drawn lines for the threshold at $\gamma=-1,-2$, and -3 . The figure shows that to detect half of the known FRBs, correcting for a redshift up to the survey limit of $500 \mathrm{pc} \mathrm{cm}^{-3}(z=0.36)$, a spectral index of -3 is required. In the bottom plot of Fig. 8, we show the rate as a function of redshift, by multiplying the volume by the rate mentioned above of 12 events per $\mathrm{Gpc}^{3}$ per day, for the three cases: all FRBs (blue line), half of the FRBs (red line), and the brightest FRB (1 out of 20, green line). From this, we see that Pilot B (blue triangle) is likely much closer to a detection than pilot A (green triangle), with approximately 16 observing beam days required, assuming a beam size of 16 square degrees and that the FRBs have a spectral index of less than -3 . For this setup with three beams, it will take 5.3 days of observing per event. We summarize this and predictions from the following sections in Table 4.

Another way to visualize observing expectations is to plot the event rate as a function of fluence, as in Fig. 7. This is shown in Fig. 9, where we plot this relation based on the volume rate of 12 events per $\mathrm{Gpc}^{3}$ per day, by calculating the luminosity distance and volume for each redshift. We have plotted vertical lines for the values corresponding to the redshift up to a DM of $110 \mathrm{pc} \mathrm{cm}^{-3}$ and $500 \mathrm{pc} \mathrm{cm}^{-3}$. We show again, as in Fig. 7, the rates versus fluence of the different surveys, as well as our rates, but now for different spectral indices. The expected rate up to a $\mathrm{DM}$ of $500 \mathrm{pc} \mathrm{cm}^{-3}$ is 154 events per sky per day, above a fluence of $6 \mathrm{kJy} \mathrm{ms}$. Given the current limits, using the same setup, a factor of nine more observing time is required, as well as a spectral index of less than -3 to see a single FRB. If the spectral index is less steep, or if only a small fraction of FRBs detected at $\sim 1 \mathrm{GHz}$ are observable at lower frequencies within the pulse width limits of our survey due to line-of-sight-dependent scattering, a longer observing time is required. In that case, other observing strategies may be more beneficial, as we discuss in Sect. 5.6. 

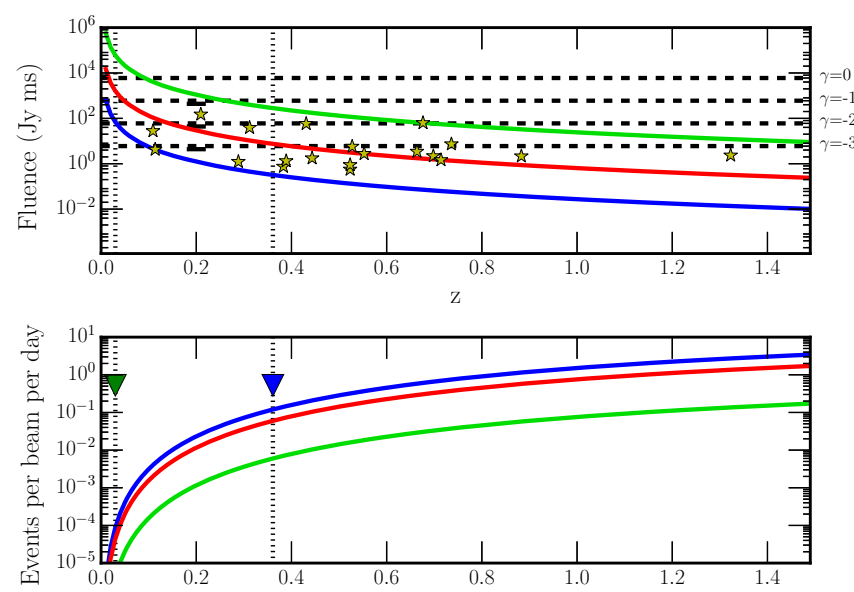

Fig. 8. Upper panel: fluence as a function of redshift for the first 20 published FRBs. Their measured values are marked by the stars. Fluence is calculated as flux density multiplied by width and the redshift is calculated as $z=\left(\mathrm{DM}_{\mathrm{FRB}}-\mathrm{DM}_{\mathrm{gal}, \mathrm{NE} 2001}-100\right) / 1100$. Values for the DMs are taken from the FRB catalog (Petroff et al. 2016). Extrapolated values are shown by the lines from top to bottom for the brightest FRB, the median FRB, and the weakest FRB in green, red, and blue, respectively. To show if they would be bright enough to be detected by LOFAR, the LOFAR fluence limit is plotted by the dotted horizontal lines for four different spectral indices $\left(v^{\gamma}\right)$. Lower panel: estimated event rate as a function of redshift. From top to bottom: for all FRBs, half of the FRBs and the "brightest" FRB (FRB160317), in blue, red, and green respectively. The redshift is limited by the DM, as indicated by the vertical dashed lines for a DM of 110 (Pilot A), and 500 (Pilot B) $\mathrm{pc} \mathrm{cm}^{-3}$. The upper limits for Pilot $\mathrm{A}$ and $\mathrm{B}$ are shown in the figure as green and blue triangles, respectively.

\subsection{Star formation history}

Hassall et al. (2013) assumes a uniform distribution of FRBs. This is the most basic assumption one can make. However, it is also possible that these events are correlated with the star formation rate (SFR), as simulated in Caleb et al. (2016b). If we use the SFR as a function of redshift, we can derive a different expected rate at the distances probed here. The rate up to a redshift $z$ is then $\mathcal{R}_{\text {sky }}(z) \sim \int_{0}^{z} \operatorname{SFR}\left(z^{\prime}\right) \frac{\mathrm{d} V_{\text {comoring }}\left(z^{\prime}\right)}{\mathrm{d} z^{\prime}} \mathrm{d} z^{\prime}$. This needs to be normalized to yield the same result of $7 \times 10^{3}$ events per sky per day up to the appropriate distance.

As an example, the Modified Salpeter A initial mass function from Table 2 of Hopkins \& Beacom (2006) is used to calculate the SFR. As the SFR is highest at $z>1$ and decreases towards $z=0$, the higher DMs (around $1000 \mathrm{pc} \mathrm{cm}^{-3}$ ) have an extra weight and fewer events are expected at lower DMs. This decreases the probability of finding FRBs at the DMs that FRATS is searching at. The expected rate including the SFR correction is plotted as the lower line in Fig. 9. At a DM of $500 \mathrm{pc} \mathrm{cm}^{-3}$, the rate is decreased by a factor 3.6 compared to a uniform distribution, requiring 58 observing beam days per event for the nonscattering case and for $\gamma<-3$.

\subsection{Local population}

Masui et al. (2015) and Kulkarni et al. (2015) discussed a large contribution to the DM from surrounding material for the case of two FRBs. In this case, the DM is not a direct measure for the distance of the bursts and the expected rates should be adjusted for that. In the extreme case, the difference in DM for the different FRBs is all from the surrounding material, and therefore all FRBs are local. In this case, the expected rate up to a DM of $500 \mathrm{pc} \mathrm{cm}^{-3}$ scales with the fraction of bursts that have a DM below $500 \mathrm{pc} \mathrm{cm}^{-3}$. Out of 29 FRBs, 6 have a DM below $500 \mathrm{pc} \mathrm{cm}^{-3}$, so we could expect about a fifth of the bursts to be visible up to a DM of $500 \mathrm{pc} \mathrm{cm}^{-3}$, provided they are bright enough.

In this case, there is no correction on the flux density for FRBs with a lower DM (or $z$ ), as was used in Sect. 5.3. Such a relationship is also not directly evident from Fig. 8 . Keane \& Petroff (2015) reported a rate of 2500 events per sky per day at a fluence of $\mathcal{F}>2 \mathrm{Jy} \mathrm{ms}$. This is also the median fluence of the known bursts. Therefore, below a DM of $500 \mathrm{pc} \mathrm{cm}^{-3}$ the rate is 500 events per sky per day.

From Table 2 we note that at $3.3 \mathrm{kJy}$ ms the limit set is 2240 events per sky per day, a factor 4.4 away from the expected rate. At this energy threshold, a spectral index of -3.2 is required, as well as 18 observing beam days per event.

\subsection{Outlook}

In this section we give an outlook on what can be achieved with LOFAR, discussing a few different strategies. LOFAR can be used in different setups. We discuss the incoherent Stokes mode $^{5}$, the coherent Stokes mode, the Fly's Eye mode (see van Haarlem et al. 2013), and an external trigger mode. The predictions are summarized in Table 4 . The general strategy here is to determine which fluence can be reached with a certain setup and derive from this an upper limit on the rate in events per sky per day given by a luminosity function $\mathcal{R}(v, \mathcal{F}) \propto v^{\gamma} \mathcal{F}^{\alpha}$. The relation between fluence and rate is taken from Fig. 7 for $(\alpha=-1, \gamma=0)$ and $(\alpha=-3 / 2, \gamma=-1.1)$. Using the total instantaneous sky coverage for a given setup, we can translate this into the number of days required for a single detection, or determine how many events will be detected in, for example, a two-year campaign.

We note that in these calculations we assume that scattering does not play a large role; see Sect. 3.3 for a discussion. If pulses are smeared out to, for example, $128 \mathrm{~ms}$, the detection threshold increases and the corresponding rate goes with $\propto \mathcal{F}^{\alpha}$. For $\alpha=$ -1 , the rate for $128 \mathrm{~ms}$ is a factor four lower and for $\alpha=-3 / 2$ it is a factor eight lower than the numbers for the duration of $8 \mathrm{~ms}$ calculated here.

\subsubsection{Commensal incoherent Stokes}

One option is to do commensal observing during the normal LOFAR cycles using incoherent Stokes beams. The detectable rate depends on the fluence and thus on the sensitivities that can be reached. The sensitivity can be improved with respect to the pilot surveys in various ways. The improvement factor on fluence is shown between parentheses. The improvements are as follows: using narrower frequency channels $(3 \mathrm{kHz})$ to counter the effects of intra-channel DM smearing and increasing the dedispersion efficiency to $90 \%$ by using small enough DM steps $(\times 3.3)$, better calibration to reach the theoretical sensitivity $(\times 2.5)$, increasing the number of (sub-)stations from 12 to $62(\times 2.2)$, and increasing the bandwidth to three sub-bands of $16 \mathrm{MHz}(\times 1.6)$. After implementing all these improvements, one can expect a total gain of a factor 30 , giving a typical fluence threshold of $100 \mathrm{Jy}$ ms based on a $3 \mathrm{kJy}$ ms typical fluence from Pilot B. For this threshold, the expected rate is eight events per sky per day for both $\alpha=-1$ and $\alpha=-3 / 2$. Using all LOFAR stations, and two beams per station, each with a beam size of $40 \mathrm{deg}^{2}$ at an average elevation of $60 \mathrm{deg}$, on average 129 days of

5 The incoherent stokes mode was previously referred to as incoherent array beam, but we now refer to the LOFAR naming of the sub-modes. 
Table 4. Rate predictions for different LOFAR observing modes and population modes.

\begin{tabular}{|c|c|c|c|c|c|c|c|c|c|c|}
\hline FRB Population Model & Stations & Mode & beams & $\begin{array}{r}\text { Predicted Rate } \\
\left(\text { sky }^{-1} \text { day }^{-1}\right)\end{array}$ & $\begin{array}{c}\mathrm{DM} \\
\left(\mathrm{pc} \mathrm{cm}^{-3}\right)\end{array}$ & $\begin{array}{l}\text { Spectral } \\
\text { index }\end{array}$ & $\begin{array}{l}\text { Req. obs. time } \\
\text { (days event }^{-1} \text { ) }\end{array}$ & $\begin{array}{l}\text { Min. Fluence } \\
\text { (Jy ms) }\end{array}$ & Section & Fig. \\
\hline Volume rate & 12 & IS & 3 & $12^{a}$ & $0-500$ & -3 & 5.3 & 6000 & 5.3 & 8,9 \\
\hline Volume rate $\mathrm{SFH}$ & 12 & IS & 3 & $3.3^{a}$ & $0-500$ & -3 & 19.3 & 6000 & 5.4 & 9 \\
\hline Local population & 12 & IS & 3 & 500 & $0-500$ & -3.2 & 6 & 3300 & 5.5 & 9 \\
\hline Caleb et al. (2017); $\alpha=-1$ & 62 & IS & 2 & 8 & - & 0 & 150 & 100 & 5.6 .1 & 7 \\
\hline Caleb et al. (2017); $\alpha=-3 / 2$ & 62 & IS & 2 & 8 & - & -1.1 & 150 & 100 & 5.6 .1 & 7 \\
\hline Caleb et al. (2017); $\alpha=-1$ & 12 & $\mathrm{CS}$ & $>100$ & 44 & - & 0 & 292 & 18 & 5.6 .2 & 7 \\
\hline Caleb et al. (2017); $\alpha=-3 / 2$ & 12 & $\mathrm{CS}$ & $>100$ & 104 & - & -1.1 & 112 & 18 & 5.6 .2 & 7 \\
\hline Caleb et al. (2017); $\alpha=-1$ & 62 & FE & 6 & 0.7 & - & 0 & 24 & 788 & 5.6 .3 & 7 \\
\hline Caleb et al. (2017); $\alpha=-3 / 2$ & 62 & $\mathrm{FE}$ & 6 & 0.4 & - & -1.1 & 10 & 1600 & 5.6 .3 & 7 \\
\hline Caleb et al. (2017); $\alpha=-1$ & 48 & TBB & - & 200 & $0-2000+$ & 0 & - & 2.5 & 5.6 .4 & \\
\hline Caleb et al. (2017); $\alpha=-1$ & 62 & TBB & - & 200 & $0-2000+$ & 0 & - & 1.5 & 5.6 .4 & \\
\hline
\end{tabular}

Notes. The columns denote the population model, as mentioned in the text, the number of LOFAR sub-stations used, the number of beams per observation, the predicted rate per sky per day for the minimum fluence, the DM range searched, the spectral index of the FRB, the required observing time per event, the given rate and the sky coverage for this setup, the minimum fluence for the given setup, and the section and the figure this model and setup refers to. The modes stand for Incoherent Stokes (IS), Coherent Stokes (CS), Fly's Eye (FE) and a Transient buffer board only mode (TBB), as explained in the corresponding sections. ${ }^{(a)}$ Events per $\mathrm{Gpc}^{3}$ per day.

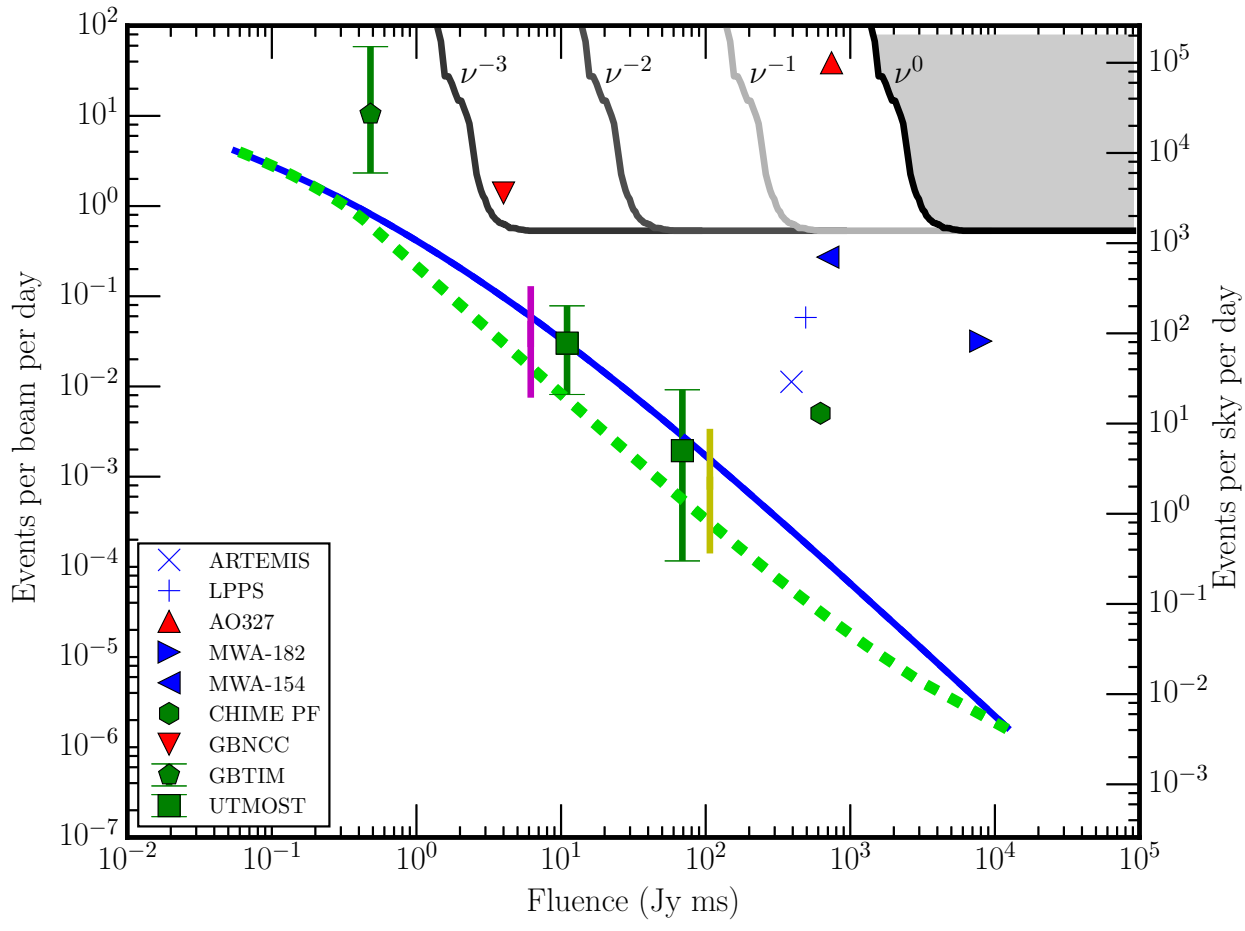

Fig. 9. Event rate vs. fluence for different surveys, corrected for a pulse length of $8 \mathrm{~ms}$. Limits from the results of Pilot B are presented by lines in the upper-right corner for spectral indices of $0,-1,-2$, and -3 . The symbols represent rate (pentagram and cubes) and upper limits for other instruments observing below $1 \mathrm{GHz}$, where green symbols observe around $800 \mathrm{MHz}$, red symbols around $350 \mathrm{MHz}$, and blue symbols below $200 \mathrm{MHz}$. Survey or telescope name, mid-frequency, DM range, and references are given in Table 3. The blue line shows event rate as a function of fluence based on a fixed rate per $\mathrm{Gpc}^{3}$, relating luminosity distance and volume as function of redshift, as explained in Sect. 3; the green dashed line shows the same but taking into account star formation history. The fluence limits for distances corresponding to DM values are indicated by the vertical lines for 110 (yellow, right) and $500 \mathrm{pc} \mathrm{cm}^{-3}$ (magenta). integration time are required to detect one event at this rate; equal to almost $3200 \mathrm{~h}$. LOFAR currently offers $1600 \mathrm{~h}$ of observing per semester. Assuming that half of the observations have a setup compatible with ours, there are 3200 observing hours in 2 years, or an overall duty cycle of $20 \%$. Thus, we expect to detect approximately one event every 2 years of commensal observing, if there is no significant loss of detectable pulses at low frequencies due to scattering and all events above threshold have a DM in the range searched.

\subsubsection{Commensal coherent Stokes}

A second option is to do commensal observing with coherent Stokes beams, improving the sensitivity. The downside is that the beam size decreases, such that many more beams, and thus much more processing power, are required for the analysis. On the positive side, this already provides an initial location which opti- mizes the search for a precise location with the TBB data. Using coherent beams, the sensitivity gain for 48 core sub-stations over 12 incoherently summed sub-stations is a factor of 13.8 . This brings down the typical fluence limit to $18 \mathrm{Jy} \mathrm{ms}$. The sky coverage decreases to $4 \mathrm{deg}^{2}$, which after two years of commensal observing gives a total of 0.0125 (1/80) sky days observed. The predicted event rate for this fluence limit is 44 or 104 events per sky per day, for the luminosity functions $(\alpha=-1, \gamma=0)$ and $(\alpha=-3 / 2, \gamma=-1.1)$, respectively. Therefore, there is a chance of $50 \%$ to detect an FRB with this strategy over a period of 2 years if $\alpha=-1$ and one event is expected if $\alpha=-3 / 2$.

\subsubsection{Fly's Eye observations}

A third option is to optimize the sky coverage by tiling out the sky in a Fly's Eye mode, using only one station per beam and a lot of beams per station with less bandwidth, with each station 
pointing in a different direction. In this case dedicated observing time is needed, so instead of finding an event rate per 2 years of commensal observing, we instead determine the amount of dedicated observing time required. We derive the numbers from the calculation in Sect. 5.6.1. Using one station instead of 62, the detection threshold is increased by a factor $\sqrt{62}$ to a fluence of $788 \mathrm{Jy} \mathrm{ms}$, while the instantaneous sky coverage increases by a factor of 62 to $2480 \mathrm{deg}^{2}$ or 0.06 sky. If we further decrease the bandwidth per sub-band to $4 \mathrm{MHz}$ instead of 16 , the detection threshold increases by a factor of $\sqrt{4}$ to $1.6 \mathrm{kJy} \mathrm{ms}$, while the sky coverage increases by a factor of 4 to 9920 square degrees, or half the visible sky instantaneously. The rate at $788 \mathrm{Jy} \mathrm{ms}$, for $\alpha=-1$ is $\sim 0.7$ events per sky per day, and at $1.6 \mathrm{kJy} \mathrm{ms}$ this is 0.4 events per sky per day. Because of the large sky coverage, this requires only 24 days and 10 days of observing for these two respective options. If such bright FRBs exist, this is a favorable observing strategy for LOFAR but requires dedicated compute power and observations, and cannot be commensally observed. It will also be difficult to find positions using the TBB data because the tile beams of all stations are pointed in different directions.

\subsubsection{External trigger}

To detect and localize FRBs with the TBB data there is another possibility, namely to trigger on FRBs at higher frequencies (e.g., 1.4 GHz). The single antenna buffers can then be read out at the time the FRB arrives at the lower frequencies minutes after the trigger. However, this requires the pulse to be detectable and an accurate DM estimate determined within the time delay given, otherwise the pulse might be missed in the TBB buffers. This was tested using the Effelsberg 100-m Radio Telescope to trigger on a known pulsar (Houben 2016) and on the FRB repeater: FRB121102 (Houben et al. 2018) by means of a specialised VOEvent standard (Petroff et al. 2017).

The buffered data can then be used to localize the pulse, a factor 100 more accurate than the Effelsberg detection. The benefit then is the certainty that there is an FRB in the data, such that all stations can be added coherently to increase the sensitivity compared to the real-time surveys. Additionally, coherent dedispersion can be applied, so intra-channel DM smearing is not an issue. For an $80 \mathrm{MHz}$ bandwidth around $150 \mathrm{MHz}^{6}$, the fluence limit at a 5 sigma level at zenith is $2.5 \mathrm{Jy} \mathrm{ms}$ for core stations or $1.5 \mathrm{Jy} \mathrm{ms}$ if all Dutch stations can be combined fully coherently. At that threshold, more than half of the known FRBs up to a DM of $500 \mathrm{pc} \mathrm{cm}^{-3}$ would be bright enough to be detected. This may be the most promising route to observe FRBs with LOFAR, since it would be possible to detect flat spectrum FRBs at $150 \mathrm{MHz}$.

\section{Conclusions}

The FRATS project searches in real-time for millisecond-scale radio transients with the LOFAR radio telescope. Some known transients of this kind are FRBs and RRATs. The focus is to obtain an optimal sky coverage by using commensal observations and a large field of view. An innovative addition is the use of ring buffers that hold the last seconds of data from each individual antenna which ultimately allows for post-event beamforming and imaging. This increases the positional accuracy, potentially to the full arc second resolution. This is important for pinpointing the location of FRBs.

\footnotetext{
6 This will be made available in a new firmware update that is currently being commissioned.
}

The potential of LOFAR is demonstrated by two different results. The first demonstration is the successful commensal observing from the Pilot $\mathrm{C}$ survey, where beamformed data were produced to search for millisecond-scale radio transients, during regular interferometric observations. Five pulsars were discovered in a 24-h scan. The second is the identification of a transient as a type II solar radio burst by localizing it in a side lobe. This could only be done by using the buffered data to create an all sky image.

Besides these demonstrations we showed the results from the pilot surveys A and B, which searched up to a DM of $\sim 110 \mathrm{pc} \mathrm{cm}^{-3}$ and $500 \mathrm{pc} \mathrm{cm}^{-3}$, respectively. While known pulsars were rediscovered, no unknown single-pulse sources have been detected. We have derived limits on the local FRB rates from these surveys.

The first pilot survey (A) had an effective observing time of 68.51 beam h, observed from 119 to $151 \mathrm{MHz}$, the lowest frequency range on which FRBs have been searched. Taking into account the pointing direction and noise level for each observation, this sets a limit on the occurrence of FRBs of less than 1500 events per sky per day above a threshold of $1.6 \mathrm{kJy} \mathrm{ms}$ below a $\mathrm{DM}$ of $\sim 110 \mathrm{pc} \mathrm{cm}^{-3}$. Pilot study B had an effective observing time of 91.2 beam $h$ in the same band. This sets limits at 1400 events per sky per day or 134 events per $\mathrm{Gpc}^{3}$ per day at a fluence threshold of $6.0 \mathrm{kJy} \mathrm{ms}$ for an 8 -ms pulse.

The expected rate is one per beam per 16 days, for a uniform extragalactic distribution, provided the source flux density follows a steep spectrum of $\sim v^{-3}$. In the case where the source population as a function of redshift follows the star formation rate, the rate drops to one per beam every 58 days. If the spectrum is less steep and/or a significant fraction of FRBs are broadened by scattering beyond our detection limits, a longer integration time is required. To be able to obtain the required observing time, we have tested commensal observing with imaging observations in an additional pilot survey (C) of $24 \mathrm{~h}$, using four $2-\mathrm{MHz}$ bands between 120 and $185 \mathrm{MHz}$. The detection of five known pulsars during this last survey validates the possibility of such commensal observations.

The future prospects are given, using as input the relations found by the UTMOST survey (Caleb et al. 2017), for a spectral index of 0 or -1 . A two-year commensal survey, assuming a realistic $20 \%$ duty cycle, would detect one event if pulse-broadening due to scattering plays no significant role. The lack of scatter broadening is not a trivial assumption at such low frequencies and high DMs, but for extragalactic bursts, it may still hold true (Lorimer et al. 2013). A dedicated survey could detect one event every 10-24 days in a Fly's Eye mode, but localization using the ring buffer data is not possible for this case. For the latter, it is favorable to use a detection at a higher frequency, and use the dispersive delay to trigger LOFAR in time. This has the benefit of using coherent beams and lowering the detection threshold to $2.5 \mathrm{Jy} \mathrm{ms}$ for a coherent core, and has the highest potential to even detect flat spectrum FRBs. Current efforts are pursuing this route, both with the Effelsberg 100-m Radio Telescope and the Westerbork Synthesis Radio Telescope (Maan \& van Leeuwen 2017), rather than continuing LOFAR-only surveys.

Acknowledgements. We thank Cees Bassa, Evan Keane, Daniele Michilli and Maura Pilia for useful discussions and Anya Bilous for her help with observing. We acknowledge funding for this work from an Advanced Grant of the European Research Council under the European Union's Seventh Framework Program (FP/2007-2013)/ERC Grant Agreement n. 227610. RPB acknowledges funding from the European Research Council (ERC) under the European Union's Horizon 2020 research and innovation programme (ERC Starting Grant agreement 715051 "Spiders"). SC acknowledges financial support from the UnivEarthS 
Labex program of Sorbonne Paris Cité (ANR-10-LABX-0023 and ANR-11IDEX-0005-02). JvL acknowledges funding from the European Research Council under the European Union's Seventh Framework Programme (FP/20072013)/ERC Grant Agreement n. 617199. This paper is based (in part) on data obtained with the International LOFAR Telescope (ILT) under project codes LC0_034, LC1_033 and LC1_052. LOFAR (van Haarlem et al. 2013) is the LOw Frequency ARray designed and constructed by ASTRON. It has observing, data processing, and data storage facilities in several countries, that are owned by various parties (each with their own funding sources), and that are collectively operated by the ILT foundation under a joint scientific policy. The ILT resources have benefitted from the following recent major funding sources: CNRSINSU, Observatoire de Paris and Université d'Orléans, France; BMBF, MIWFNRW, MPG, Germany; Science Foundation Ireland (SFI), Department of Business, Enterprise and Innovation (DBEI), Ireland; NWO, The Netherlands; The Science and Technology Facilities Council, UK; Ministry of Science and Higher Education, Poland.

\section{References}

Amiri, M., Bandura, K., Berger, P., et al. 2017, ApJ, 844, 161

Bannister, K. W., Shannon, R. M., Macquart, J.-P., et al. 2017, ApJ, 841, L12

Bassa, C. G., Tendulkar, S. P., Adams, E. A. K., et al. 2017, ApJ, 843, L8

Bhat, N. D. R., Cordes, J. M., Camilo, F., Nice, D. J., \& Lorimer, D. R. 2004, ApJ, 605, 759

Bonsignori-Facondi, S. R., Salter, C. J., \& Sutton, J. M. 1973, A\&A, 27, 67

Boyle, P. C., \& CHIME/FRB Collaboration 2018, ATel, 11901

Buitink, S., Corstanje, A., Falcke, H., et al. 2016, Nature, 531, 70

Burke-Spolaor, S. 2013, IAU Symp., 291, 95

Burke-Spolaor, S., \& Bailes, M. 2010, MNRAS, 402, 855

Burke-Spolaor, S., Bailes, M., Johnston, S., et al. 2011, MNRAS, 416, 2465

Caleb, M., Flynn, C., Bailes, M., et al. 2016a, MNRAS, 458, 718

Caleb, M., Flynn, C., Bailes, M., et al. 2016b, MNRAS, 458, 708

Caleb, M., Flynn, C., Bailes, M., et al. 2017, MNRAS, 468, 3746

Champion, D. J., Petroff, E., Kramer, M., et al. 2016, MNRAS, 460, L30

Chatterjee, S., Law, C. J., Wharton, R. S., et al. 2017, Nature, 541, 58

Chawla, P., Kaspi, V. M., Josephy, A., et al. 2017, ApJ, 844, 140

Coenen, T. 2013, PhD Thesis, University of Amsterdam, http://dare.uva. nl/en/record/459730

Coenen, T., van Leeuwen, J., Hessels, J. W. T., et al. 2014, A\&A, 570, A60

Connor, L., Lin, H.-H., Masui, K., et al. 2016, MNRAS, 460, 1054

Cordes, J. M., \& Lazio, T. J. W. 2002, ArXiv e-prints [astro-ph/0207156]

Cordes, J. M., Weisberg, J. M., \& Boriakoff, V. 1985, ApJ, 288, 221

Cunha-Silva, R. D., Fernandes, F. C. R., \& Selhorst, C. L. 2015, A\&A, 578, A38

Dai, Z. G., Wang, J. S., Wu, X. F., \& Huang, Y. F. 2016, ApJ, 829, 27

Davies, J. G., \& Large, M. I. 1970, MNRAS, 149, 301

Deneva, J. S., Cordes, J. M., McLaughlin, M. A., et al. 2009, ApJ, 703, 2259

Deneva, J. S., Stovall, K., McLaughlin, M. A., et al. 2016, ApJ, 821, 10

Deng, W., \& Zhang, B. 2014, ApJ, 783, L35

Edwards, R. T., Bailes, M., van Straten, W., \& Britton, M. C. 2001, MNRAS, 326,358

Egorov, A. E., \& Postnov, K. A. 2009, Astron. Lett., 35, 241

Ershov, A. A., \& Kuzmin, A. D. 2003, Astron. Lett., 29, 91

Falcke, H., \& Rezzolla, L. 2014, A\&A, 562, A137
Fender, R., Wijers, R., Stappers, B., \& LOFAR Transients Key Science Project 2008, Proc. Science 2007, PoS Dynamic, 030, 2007

Geng, J. J., \& Huang, Y. F. 2015, ApJ, 809, 24

Hamaker, J. P. 2011, Tech. Rep. ASTRON

Hansen, B. M. S., \& Lyutikov, M. 2001, MNRAS, 322, 695

Haslam, C. G. T., Salter, C. J., Stoffel, H., \& Wilson, W. E. 1982, A\&AS, 47, 1

Hassall, T. E., Keane, E. F., \& Fender, R. P. 2013, MNRAS, 436, 371

Hogg, D. W. 1999, ArXiv e-prints [astro-ph/9905116]

Hopkins, A. M., \& Beacom, J. F. 2006, ApJ, 651, 142

Houben, L. J. M. 2016, Master's Thesis, Radboud University Nijmegen

Houben, L. J. M., Spitler, L. G., ter Veen, S., et al. 2018, A\&A, submitted

Ioka, K. 2003, ApJ, 598, L79

Karastergiou, A., Chennamangalam, J., Armour, W., et al. 2015, MNRAS, 452 , 1254

Karuppusamy, R., Stappers, B. W., \& Serylak, M. 2011, A\&A, 525, A55

Keane, E. F., \& Petroff, E. 2015, MNRAS, 447, 2852

Keane, E. F., Kramer, M., Lyne, A. G., Stappers, B. W., \& McLaughlin, M. A. 2011, MNRAS, 415, 3065

Kulkarni, S. R., Ofek, E. O., Neill, J. D., Zheng, Z., \& Juric, M. 2014, ApJ, 797, 70

Kulkarni, S. R., Ofek, E. O., \& Neill, J. D. 2015, ArXiv e-prints [arXiv:1511.09137]

Law, C. J., Abruzzo, M. W., Bassa, C. G., et al. 2017, ApJ, 850, 76

Lawson, K. D., Mayer, C. J., Osborne, J. L., \& Parkinson, M. L. 1987, MNRAS, 225,307

Lorimer, D. R., Bailes, M., McLaughlin, M. A., Narkevic, D. J., \& Crawford, F. 2007, Science, 318, 777

Lorimer, D. R., Karastergiou, A., McLaughlin, M. A., \& Johnston, S. 2013, MNRAS, 436, L5

Maan, Y., \& van Leeuwen, J. 2017, Proc. URSI GASS 2017, in press [arXiv:1709.06104]

Marcote, B., Paragi, Z., Hessels, J. W. T., et al. 2017, ApJ, 834, L8

Maron, O., Kijak, J., Kramer, M., \& Wielebinski, R. 2000, A\&AS, 147, 195

Masui, K., Lin, H.-H., Sievers, J., et al. 2015, Nature, 528, 523

McLaughlin, M. A., Lyne, A. G., Lorimer, D. R., et al. 2006, Nature, 439, 817

McQuinn, M. 2014, ApJ, 780, L33

Miller, J., McLaughlin, M., Rea, N., et al. 2011, AIP Conf. Ser., 1357, 161

Mottez, F., \& Zarka, P. 2014, A\&A, 569, A86

Petroff, E., Barr, E. D., Jameson, A., et al. 2016, PASA, 33, e045

Petroff, E., Houben, L., Bannister, K., et al. 2017, ArXiv e-prints [arXiv:1710.08155]

Planck Collaboration XVI. 2014, A\&A, 571, A16

Popov, S. B., \& Postnov, K. A. 2007, ArXiv e-prints [arXiv:0710. 2006]

Popov, S. B., \& Postnov, K. A. 2013, ArXiv eprints [arXiv:1307 .4924]

Rees, M. J. 1977, Nature, 266, 333

Rowlinson, A., Bell, M. E., Murphy, T., et al. 2016, MNRAS, 458, 3506

Schellart, P., Nelles, A., Buitink, S., et al. 2013, A\&A, 560, A98

Spitler, L. G., Cordes, J. M., Hessels, J. W. T., et al. 2014, ApJ, 790, 101

Tendulkar, S. P., Bassa, C. G., Cordes, J. M., et al. 2017, ApJ, 834, L7

ter Veen, S. 2015, PhD Thesis, Radboud University Nijmegen, http://hdl. handle.net/2066/147186

Thornton, D., Stappers, B., Bailes, M., et al. 2013, Science, 341, 53

Tingay, S. J., Trott, C. M., Wayth, R. B., et al. 2015, AJ, 150, 199

van Haarlem, M. P., Wise, M. W., Gunst, A. W., et al. 2013, A\&A, 556, A2

Wijnholds, S., \& van Cappellen, W. 2011, IEEE Trans. Antennas Propag., 59, 1981

Xu, J., \& Han, J. L. 2015, Res. Astron. Astrophys., 15, 1629 